\title{
Ryanodine receptor-bound calmodulin is essential to protect against catecholaminergic polymorphic ventricular tachycardia
}

\author{
Yoshihide Nakamura, ${ }^{1}$ Takeshi Yamamoto, ${ }^{2}$ Shigeki Kobayashi, ${ }^{1}$ Masaki Tamitani, ${ }^{1}$ \\ Yoriomi Hamada, ${ }^{1}$ Go Fukui, ${ }^{1}$ Xiaojuan $\mathrm{Xu},{ }^{3}$ Shigehiko Nishimura, ${ }^{1}$ Takayoshi Kato, \\ Hitoshi Uchinoumi, ${ }^{1}$ Tetsuro Oda, ${ }^{1}$ Shinichi Okuda, ${ }^{1}$ and Masafumi Yano ${ }^{1}$ \\ 'Department of Medicine and Clinical Science, Division of Cardiology, and 'Faculty of Health Sciences, Yamaguchi \\ University Graduate School of Medicine, Yamaguchi, Japan. ${ }^{3}$ Department of Pathology and Pathophysiology, School of \\ Medicine, Tongji University, Shanghai, China.
}

\begin{abstract}
Catecholaminergic polymorphic ventricular tachycardia (CPVT) is caused by a single point mutation in the cardiac type 2 ryanodine receptor (RyR2). Using a knockin (KI) mouse model (R2474S/+), we previously reported that a single point mutation within the RyR2 sensitizes the channel to agonists, primarily mediated by defective interdomain interaction within the RyR2 and subsequent dissociation of calmodulin (CaM) from the RyR2. Here, we examined whether CPVT can be genetically rescued by enhancing the binding affinity of CaM to the RyR2. We first determined whether there is a possible amino acid substitution within the CaM-binding domain in the RyR2 (3584-3603 residues) that can enhance its binding affinity to CaM and found that V3599K substitution showed the highest binding affinity of CaM to the CaM-binding domain. Hence, we generated a heterozygous $\mathrm{KI}$ mouse model (V3599K/+) with a single amino acid substitution in the CaM-binding domain of the RyR2 and crossbred it with the heterozygous CPVT-associated R2474S/+-KI mouse to obtain a double-heterozygous R2474S/V3599K-KI mouse model. The CPVT phenotypes - bidirectional or polymorphic ventricular tachycardia, spontaneous $\mathrm{Ca}^{2+}$ transients, and $\mathrm{Ca}^{2+}$ sparks - were all inhibited in the R2474S/V3599K mice. Thus, enhancement of the CaMbinding affinity of the RyR2 is essential to prevent CPVT-associated arrhythmogenesis.
\end{abstract}

Authorship note: YN, TY, and SK contributed equally to this work.

Conflict of interest: The authors have declared that no conflict of interest exists.

Copyright: () 2019 American Society for Clinical Investigation

Submitted: November 13, 2018

Accepted: April 17, 2019

Published: June 6, 2019.

Reference information: /CI Insight. 2019;4(11):e126112. https://doi. org/10.1172/jci.insight.126112.

\section{Introduction}

The cardiac type 2 ryanodine receptor (RyR2) is a huge tetrameric protein in the sarcoplasmic reticulum (SR) through which a large amount of $\mathrm{Ca}^{2+}$ is released from the SR, triggered by a small amount of $\mathrm{Ca}^{2+}$ entering the L-type $\mathrm{Ca}^{2+}$ channel $(1,2)$. To date, more than 150 mutations within the RyR2 have been reported to be linked with catecholaminergic polymorphic ventricular tachycardia (CPVT) (3). The point mutations observed in CPVT are not randomly distributed but clustered into 3 hot domains: the N-terminal (1-600 residues), the central (2000-2500 residues), and the C-terminal (4). Based on the "domain switch hypothesis" Ikemoto and colleagues proposed (5), we demonstrated that the single point mutation causes unzipping between the N-terminal and the central domains, which gave rise to a $\mathrm{Ca}^{2+}$ leak in the CPVT-associated R2474S/+-knockin (R2474S/+-KI) mouse (6) and also in a canine model of tachycardia-induced heart failure (7-9). These findings suggest that domain unzipping between the $\mathrm{N}$-terminal and the central domains plays a critical role in the pathogenesis of lethal arrhythmia and heart failure as well. Recently, we found that domain unzipping displaces calmodulin ( $\mathrm{CaM}$ ) from the RyR2, which otherwise plays an inhibitory role in channel opening, causing $\mathrm{Ca}^{2+}$ leak in CPVT (10) and heart failure (11). Moreover, we found that Gly-Ser-His CaM (GSH-CaM), which has a higher binding affinity to the RyR2 than WT CaM, inhibited spontaneous $\mathrm{Ca}^{2+}$ sparks or transients as an arrhythmogenic substrate of inducing CPVT (12) and ameliorated contractile dysfunction in heart failure (13). These notions support the idea that the specific binding of CaM to the RyR2 plays a critical role in the pathogenesis of CPVT and heart failure. Furthermore, the fact that genetic modulation of the binding site of CaM to the RyR2 ( 3 amino acid substitutions: RyR2- 
Table 1. Single amino acid substitution within the CaM-binding domain in the RyR2

\begin{tabular}{|c|c|}
\hline Name & Amino acid sequence \\
\hline WT & ${ }^{3584}$ AVWHKLLSKQRKRAVVACFR ${ }^{3603}$ \\
\hline 1 & AVWHRLLLSKQ̨RKRAVVACFR \\
\hline 2 & AVWHKKYLSKQQRKRAVVACFR \\
\hline 3 & AVWHKFLSKQRKRAVVACFR \\
\hline 4 & AVWHKWLSKQRKRAVVACFR \\
\hline 5 & AVWHKILSKQRKRAVVACFR \\
\hline 6 & AVWHKQిLSKQRKRAVVACFR \\
\hline 7 & AVWHKLLSKㅌRKRAVVACFR \\
\hline 8 & AVWHKLLSKRRRKRAVACFR \\
\hline 9 & AVWHKLLSKERKRAVVACFR \\
\hline 10 & AVWHKLLSKQQRRRAVVACFR \\
\hline 11 & AVWHKLLSKQ̨RKRㅁVACFR \\
\hline 12 & AVWHKLLSKQQRKRWWVACFR \\
\hline 13 & AVWHKLLSKQRRKRQVVACFR \\
\hline 14 & AVWHKLLSKQRKRCVVACFR \\
\hline 15 & AVWHKLLSKQRKRAFVACFR \\
\hline 16 & AVWHKLLSKQRKRAYVACFR \\
\hline 17 & AVWHKLLSKQQRKRAMVACFR \\
\hline 18 & AVWHKLLSKQQRKRAQ̨VACFR \\
\hline 19 & AVWHKLLSKQ̨RKRALVACFR \\
\hline 20 & AVWHKLLSKQRKRAVEACFR \\
\hline 21 & AVWHKLLSKQQRKRAVYYACFR \\
\hline 22 & AVWHKLLSKQQRKRAVMACFR \\
\hline 23 & AVWHKLLSKQQRKRAVQ̣ACFR \\
\hline 24 & AVWHKLLSKQQRKRAVㅌACFR \\
\hline 25 & AVWHKLLSKQRKRLVVACFR \\
\hline
\end{tabular}

The underlined letters represent the substituted amino acids.

W3587A, -L3591D, -F3603A), which prevented CaM binding to the RyR2, induced premature death of the KI mice within 1 month, associated with marked cardiac enlargement and hypertrophy (14), also strongly indicates the essential role of CaM bound to the RyR2 in maintenance of cardiac function and structure. However, it remains to be elucidated as to whether forced association of CaM to the defective RyR2 rescues the diseased phenotypes. We hypothesized that the enhancement of CaM-binding affinity toward the defective RyR2 under stressful conditions prevents spontaneous $\mathrm{Ca}^{2+}$ release, thereby preventing CPVT.

\section{Results}

A single amino acid substitution (V3599K) within the CaM-binding domain in the RyR2 (3584-3603 residues) markedly enhanced the binding affinity to CaM. We first determined whether there is a possible amino acid substitution within the CaM-binding domain in the RyR2 (3584-3603 residues) that can enhance its binding affinity. For this purpose, we assessed the binding of CaM to the RyR2 using a photoreactive cross-linker, sulfosuccinimidyl-6-(4'-azido-2'-nitrophenylamino)hexanoate (sulfo-SANPAH) (see Methods) to evaluate the CaM-binding affinity of 20-mer CaM-binding peptides (CaMBPs) harboring the same amino acid sequence as the CaM-binding domain (3584-3603 residues) except 1 amino acid residue (Table 1). Of the 25 peptides, peptide 24, in which a V3599K substitution was done, showed the highest inhibition of CaM-SANPAH binding to the RyR2, indicating a higher binding affinity of CaM to the $24 \mathrm{CaMBP}$ than to the RyR2 (Figure 1). The conformational state change of CaM, upon its binding to CaMBPs, was also evaluated by measuring the tryptophan fluorescence change. As shown in Supplemental Figure 1 (supplemental material available online with this article; https://doi.org/10.1172/jci. insight.126112DS1), the V3599K CaMBP showed the highest change in CaM-mediated tryptophan fluorescence. The effect of a single amino acid substitution (V3599K) within the CaMBP on the binding affinity of CaM was also confirmed by quartz crystal microbalance (QCM) method. The single amino acid substitution in CaMBP (CaMBP mutation [CaMBPmut]: V3599K) markedly enhanced the binding of CaM to CaMBPmut. Suramin, which directly binds to the core RyR-CaM-binding sequence and displaces CaM from both RyR1 and RyR2 channel isoforms (15-17), indeed inhibited CaM binding to both WT CaMBP and CaMBPmut (Figure 2). 
A
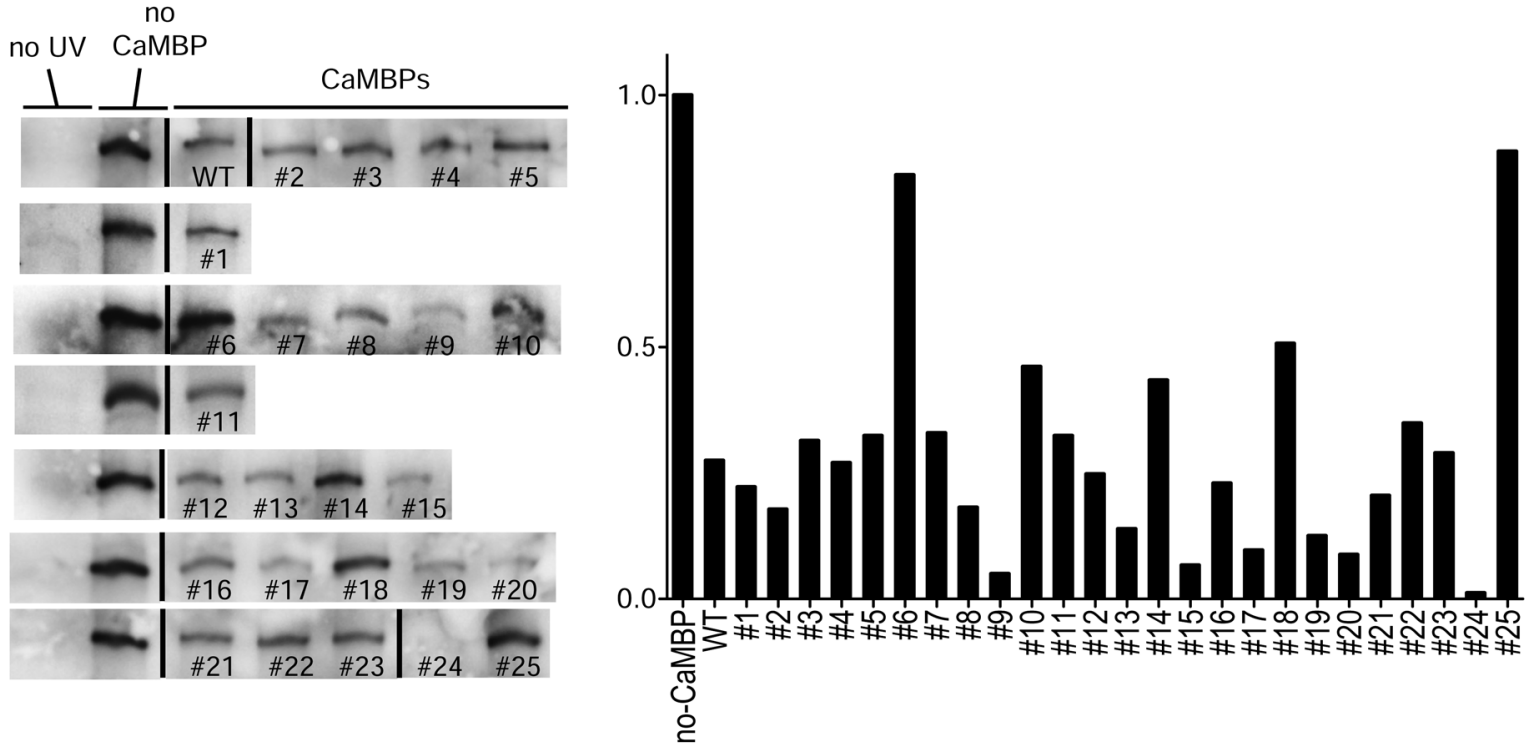

B
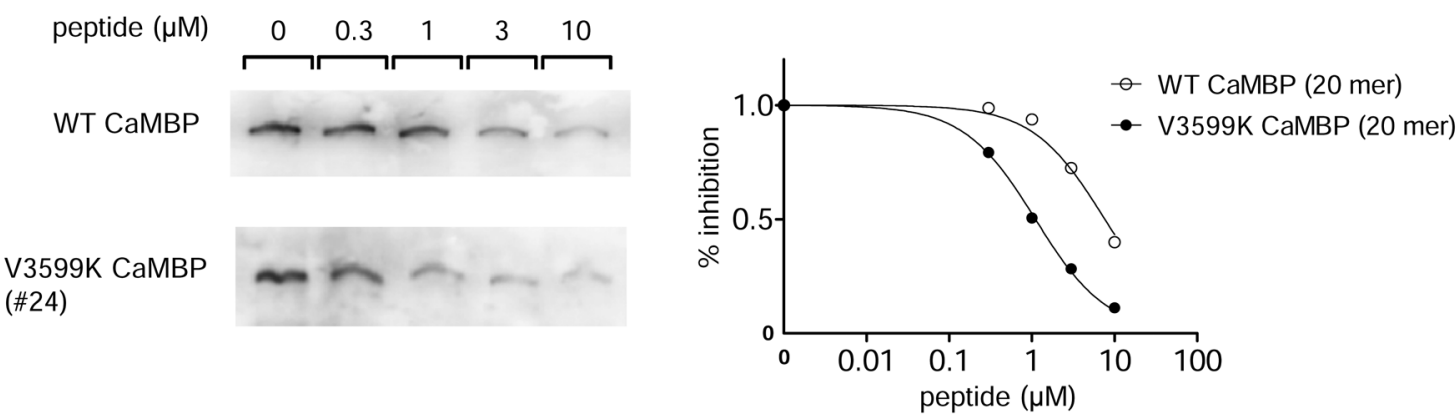

Figure 1. Determination of a single amino acid substitution within the CaM-binding domain (3584-3603 residues) in the RyR2 by which CaM-binding affinity is highly increased. (A) Evaluation of the binding affinity of 20-mer CaM-binding peptides (CaMBPs) harboring the same amino acid sequence as the CaM-binding domain (3584-3603 residues) except 1 amino acid residue by CaM-SANPAH cross-linking method (see Methods). Of 25 peptides, peptide 24, in which V3599K substitution was done, showed the highest inhibition of CaM-SANPAH binding to the RyR2, indicating a higher binding affinity of CaM to the 24 CaMBP than to the RyR2. The $y$ axis represents the ratio of the density to no CaMBP. (B) Concentration-dependent inhibitory effect of either WT CaMBP or V3599K CaMBP on CaM-SANPAH binding to the RyR2 V3599K CaMBP showed a strong inhibition compared with WT CaMBP, indicating a higher binding affinity of V3599K CaMBP to CaM than of WT CaMBP.

Ventricular tachycardia was not induced by catecholamine or exercise in a R2474S/V3599K-KI mouse model. We then generated a heterozygous V3599K/+-KI mouse model in which the binding affinity of CaM to the RyR2 was markedly enhanced by a single amino acid mutation (V3599K) at the CaM-binding domain within the RyR2 (Supplemental Figure 2), and then we crossbred the V3599K/+-KI mouse with the R2474S/+-KI mouse to obtain a heterozygous R2474S/V3599K-KI mouse model. There was no appreciable difference in the structural or functional features of the hearts among WT (+/+), V3599K/+-KI, and R2474S/V3599K-KI mice (structural features, Supplemental Figure 3; echocardiography, Supplemental Figure 4; protein expression and phosphorylation, Supplemental Figure 5).

We previously demonstrated that in the pathogenesis of CPVT, catecholamine-mediated phosphorylation of the RyR2 at serine 2808 (Ser2808) caused domain unzipping between the N-terminal and the central domains, which in turn dissociated CaM from the RyR2, inducing $\mathrm{Ca}^{2+}$ leak, leading to delayed afterdepolarization $(\mathrm{DaD})$ and finally lethal arrhythmia $(6,10)$. In this study, we examined whether arrhythmia is observed in R2474S/V3599K-KI mice using 24-hour electrocardiography (ECG) monitoring and examined the effect of epinephrine or exercise on ventricular arrhythmia. Ventricular tachycardia (VT) was easily induced by epinephrine or exercise in all R2474S/+ mice but not in R2474S/V3599K-KI mice 

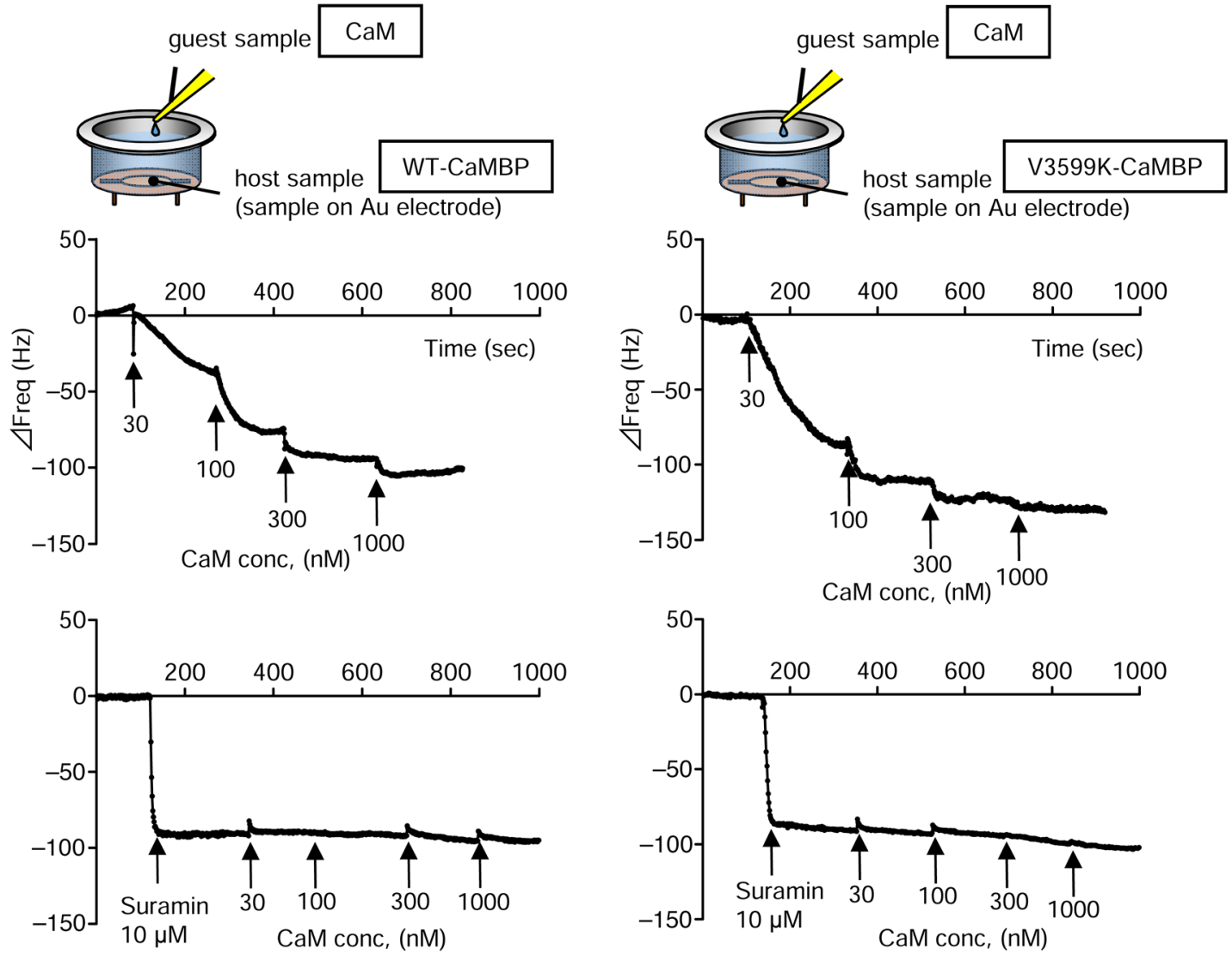

Figure 2. Evaluation of the binding affinity of CaM to either WT CaMBP (left) or V3599K CaMBP (right) by QCM. Each CaMBP was first immobilized on the sensor chip surface as a host sample, and CaM was added cumulatively in a concentration-dependent manner to evaluate the binding affinity of CaM to each CaMBP, as described in Methods. The binding affinity of CaM to V3599K CaMBP was much higher than to WT CaMBP. Suramin (10 $\mu$ M) inhibited CaM binding to both WT CaMBP and CaMBPmut.

as well as WT and V3599K/+-KI mice (Figure 3, A and B). Under 24-hour ECG monitoring, both VT and ventricular bigeminy were frequently observed even under a small amount of stress in R2474S/+ mice but not mainly in R2474S/V3599K-KI mice (Figure 3, C and D).

Spontaneous $\mathrm{Ca}^{2+}$ transients and $\mathrm{Ca}^{2+}$ sparks were both inhibited in the $\mathrm{R} 2474 \mathrm{~S} / \mathrm{V} 3599 \mathrm{~K}$ mice. There was no statistically significant difference in the contour or kinetic parameters of cell shortening (Figure 4) and $\mathrm{Ca}^{2+}$ transients (Figure 5A) at the baseline between the WT and V3599K/+-KI mice. In response to ISO, the time from the peak to an $80 \%$ decline in cell shortening or $\mathrm{Ca}^{2+}$ transients was prolonged in $\mathrm{R} 2474 \mathrm{~S} /+$ mouse cardiomyocytes, suggesting a delay in the inactivation of $\mathrm{Ca}^{2+}$ or spontaneous $\mathrm{Ca}^{2+}$ release events. However, the time from the peak to an $80 \%$ decline in cell shortening or $\mathrm{Ca}^{2+}$ transient was virtually restored in R2474S/V3599K-KI cardiomyocytes (Figure 4 and Figure 5A). In the presence of ISO, the SR $\mathrm{Ca}^{2+}$ content increased in WT and V3599K/+-KI mice, while the extent of increase decreased in R2474S/+ mice, presumably because of $\mathrm{Ca}^{2+}$ leak (Figure 5B). On the other hand, the decrease in $\mathrm{SR} \mathrm{Ca}{ }^{2+}$ content in R2474S/+-KI mice was not observed in R2474S/V3599K-KI mice (Figure 5B). In the presence of ISO, the $\mathrm{Ca}^{2+}$ spark frequency increased in R2474S/+-KI mice compared with the R2474S/V3599K-KI mice (Figure 6A). Compared with the WT cardiomyocytes, both the peak amplitude and full width at half maximum (FWHM) decreased whereas full duration at half maximum (FDHM) increased in R2474S/+-KI cardiomyocytes, suggesting a delay in the inactivation of the RyR2. In contrast, the increase in FDHM was attenuated in R2474S/V3599K-KI cardiomyocytes (Figure 6B and Table 2). In the presence of ISO, spontaneous $\mathrm{Ca}^{2+}$ transients (SCaTs) increased in R2474S/+-KI mice, while it was hardly observed in R2474S/ V3599K-KI mice (Figure 7). These findings clearly indicate that the defective interaction of CaM with the RyR2 and the subsequent $\mathrm{Ca}^{2+}$ leak causes not only an arrhythmogenic substrate leading to CPVT, but also of particular interest, a relaxation disturbance (upon PKA phosphorylation) in intact cardiomyocytes. 


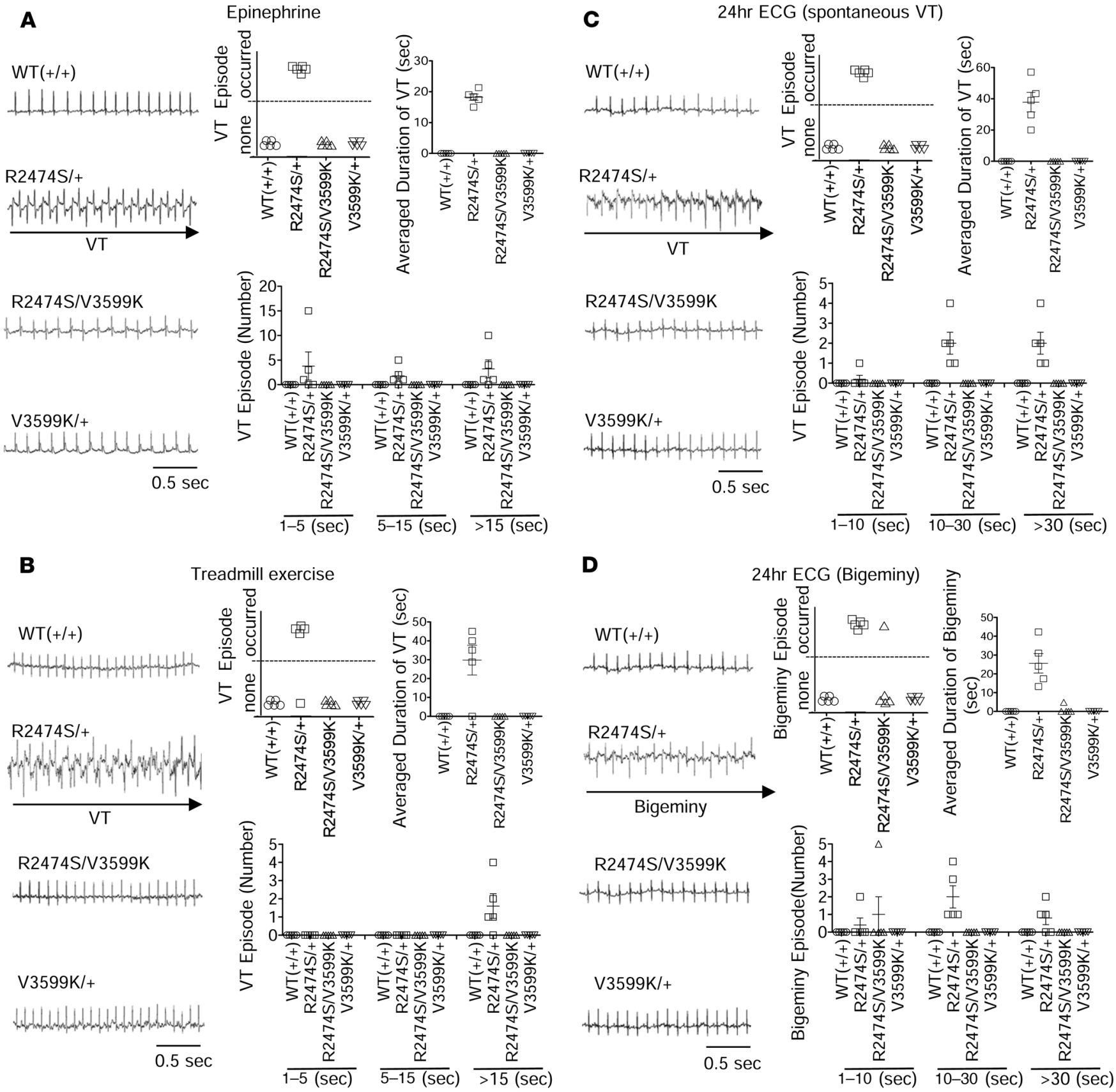

Figure 3. Inhibition of ventricular arrhythmia by V3599K mutation in mice. Representative ECG recordings (left) and the summarized data for VT induction (right) after (A) administration of epinephrine (1 mg/kg of body weight, i.p.) or (B) treadmill exercise in WT $(+/+)(n=5)$, R2474S/+-KI $(n=5)$, V3599K/+-KI $(n=5)$, and R2474S/V3599K-KI mice $(n=5)$. Representative 24-hour ECG recordings (left) and the summarized data (right) on (C) spontaneous VT or $(\mathbf{D})$ ventricular bigeminy in WT $(n=5), \mathrm{R} 2474 \mathrm{~S} /+-\mathrm{KI}(n=5)$, V3599K/+-KI $(n=5)$, and R2474S/V3599K-KI mice $(n=5)$.

To further examine the effect of V3599K mutation on the channel gating, independent of CaM, we measured the $\mathrm{Ca}^{2+}$ sparks after pretreatment with suramin $(10 \mu \mathrm{M})$ (followed by washing off), which has been shown to displace endogenous CaM (15-17), in saponin-permeabilized cardiomyocytes. First, without the suramin wash, we examined the effect of exogenously added CaM (100 nM) on the $\mathrm{Ca}^{2+}$ spark frequency. With exogenous CaM $(100 \mathrm{nM})$ added in the internal solution, there was no difference in the $\mathrm{Ca}^{2+}$ spark frequency in the absence of cAMP among all groups (Figure 8, A and B). However, in the presence of cAMP $(1 \mu \mathrm{M})$, the $\mathrm{Ca}^{2+}$ spark frequency markedly increased in R2474S/+ cardiomyocytes but not in other cardiomyocytes (Figure 8, A and B). In contrast, after treatment with suramin (and without exogenous $\mathrm{CaM}$ ), namely no CaM condition, the $\mathrm{Ca}^{2+}$ sparks were only transiently observed and quickly 


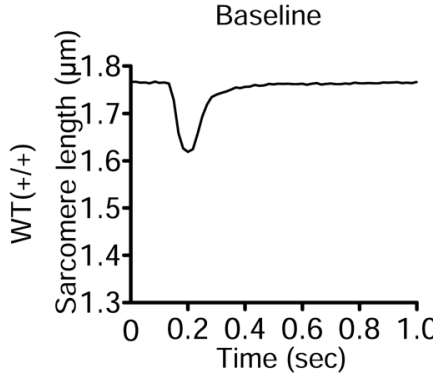

ISO
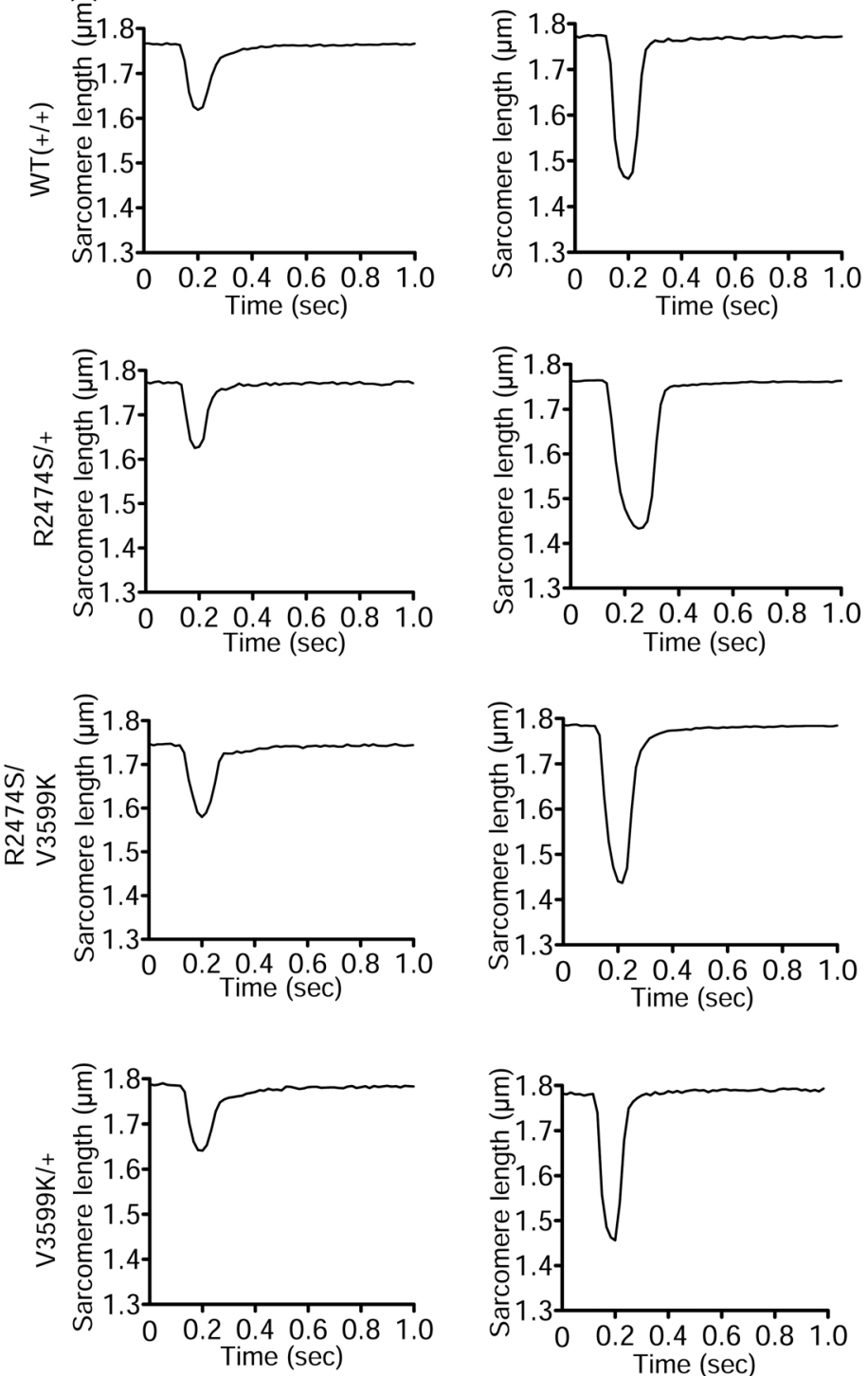
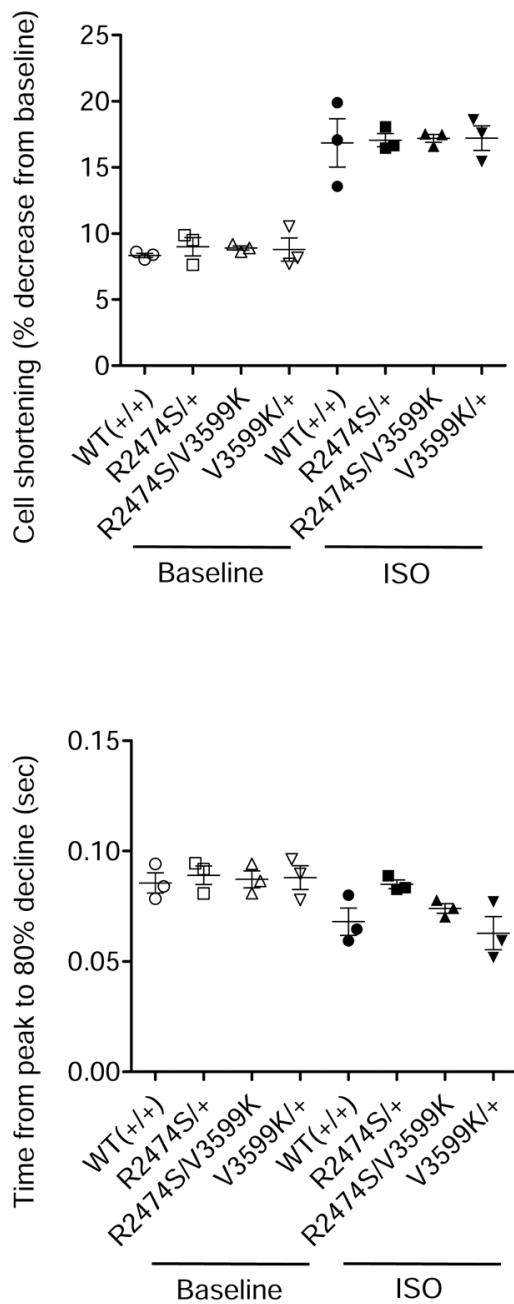

Figure 4. Cell shortening in intact cardiomyocytes. Representative recordings of sarcomere length at a pacing rate of $1 \mathrm{~Hz}$, with isoproterenol (10 $\mathrm{nM})$ activation (ISO) and without it (baseline), and the summarized data (right). Values for individual mice are plotted with mean \pm SEM; $n=27-44$ cells from 3 hearts.

disappeared in the absence of cAMP at baseline (data not shown), probably owing to the decrease in intra$\mathrm{SR}\left[\mathrm{Ca}^{2+}\right]$ caused by the leaky RyR2. However, in the presence of cAMP, the $\mathrm{Ca}^{2+}$ spark frequency markedly increased to a similar extent in all groups, without a significant change in SR $\mathrm{Ca}^{2+}$ content (Figure 8B). These results clearly indicate that the rescue effect of V3599K mutation on aberrant $\mathrm{Ca}^{2+}$ release caused by CPVT-associated mutation (R2474S) is indeed CaM dependent.

CaM binding to the RyR2 was restored in the R2474S/V3599K mice. In resting ventricular cardiomyocytes, only approximately $1 \%$ of total CaM is freely diffusible (18), and the bound CaM is highly concentrated along $\mathrm{Z}$ lines, where more than $90 \%$ of that CaM is RyR2 bound (17). Moreover, we recently found that either angiotensin II or phenylephrine caused translocation of CaM bound to the $\mathrm{Z}$ line to the nucleus with $G$ protein-coupled receptor kinase 5, and dantrolene, an RyR2 stabilizer, prevented the translocation of CaM to the nucleus (19), indicating that the reduction in $\mathrm{Z}$ line CaM binding is indicative of decreased RyR2 binding. Based on these findings, we examined CaM bound to the $\mathrm{Z}$ line along with that in the nucleus in intact cardiomyocytes to assess whether CaM binding to the RyR2 is maintained by V3599K mutation, even in the presence of ISO. In confirmation of our previous report (10), endogenous CaM was well colocalized with the RyR2 on the $\mathrm{Z}$ line (Figure 9). The $\mathrm{Z}$ line-bound CaM decreased in parallel with 
A
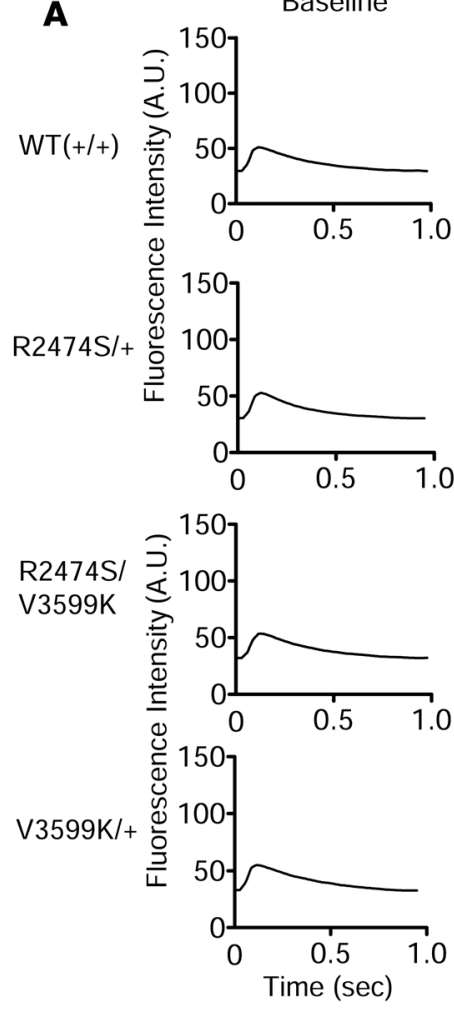

ISO
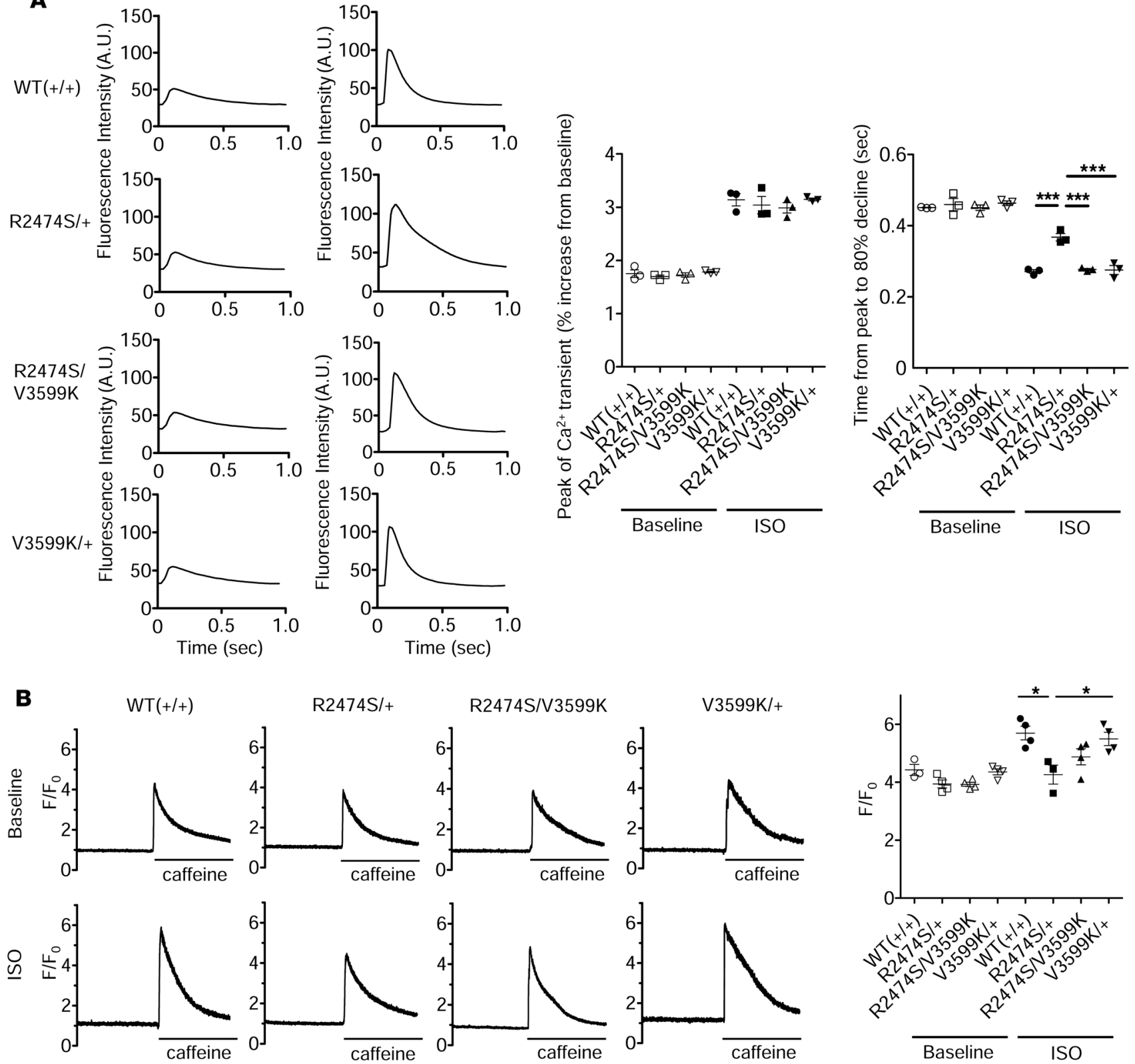

Figure 5. Intracellular $\mathrm{Ca}^{2+}$ transients and SR $\mathrm{Ca}^{2+}$ content in intact cardiomyocytes. (A) Representative recordings of fluo-4 AM fluorescence signal, at a pacing rate of $1 \mathrm{~Hz}$, with ISO (10 nM) activation (ISO) and without it (baseline), and the summarized data (right). $n=28-44$ cells from 3 hearts. (B) Representative recording of fluo-4 AM fluorescence signal after addition of caffeine, which is a measure of the SR $\mathrm{Ca}^{2+}$ content, and the summarized data (right). $n=10-19$ cells from 3 to 4 hearts. Caffeine-induced $\mathrm{Ca}^{2+}$ transients were measured by first applying a stimulation train at $2 \mathrm{~Hz}$ and then by rapidly switching from the superfusing solution to a solution containing $20 \mathrm{mM}$ caffeine for 5 to 6 seconds. Values for individual mice are plotted with mean \pm SEM. ${ }^{*} P<0.05$, and ${ }^{* *} P<0.001$ (1-way ANOVA with post hoc Tukey's multiple-comparison test).

the increase in nuclear CaM only in R2474-KI cardiomyocytes after the addition of ISO, whereas its levels were restored in R2474S/V3599K-KI cardiomyocytes (Figure 9), although the phosphorylation level at Ser2808 was similarly increased by ISO among all mice (Supplemental Figure 6). These data strongly suggest that in response to catecholamine, CaM binding to the RyR2 is restored by the copresence of V3599K mutation upon R2474S-associated channel disorder.

To further examine whether there is any difference in the kinetics of the CaM binding to the RyR2 between WT and KI hearts, we assessed the CaM binding to subcellular fractions by introducing CaM, fluorescently 
A

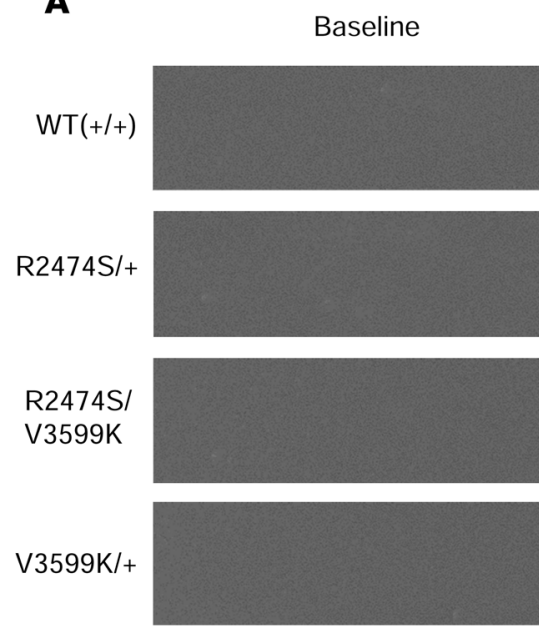

B

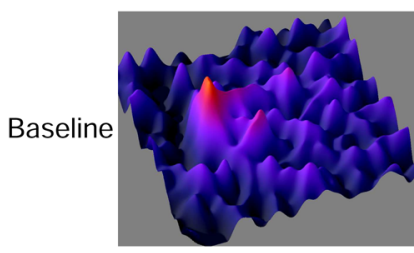

ISO

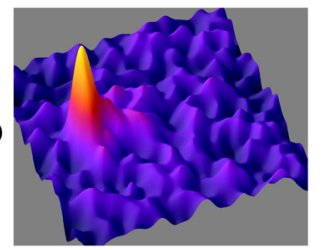

ISO
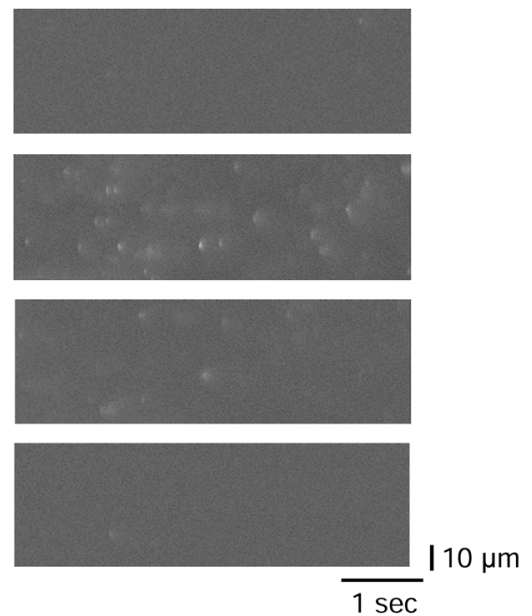

$\mathrm{R} 2474 \mathrm{~S} /+$
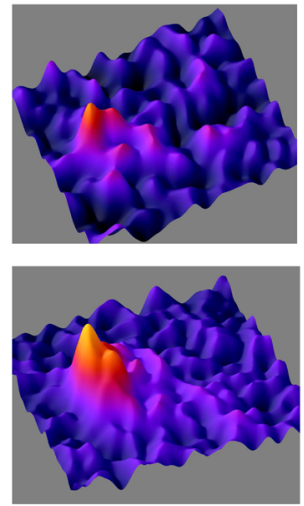

R2474S/ V3599K
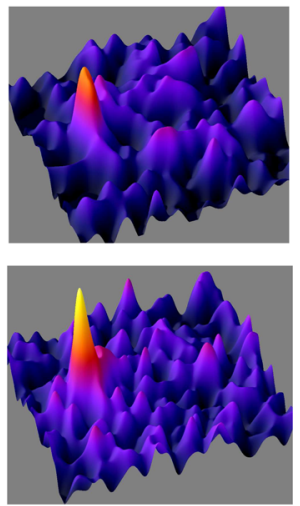

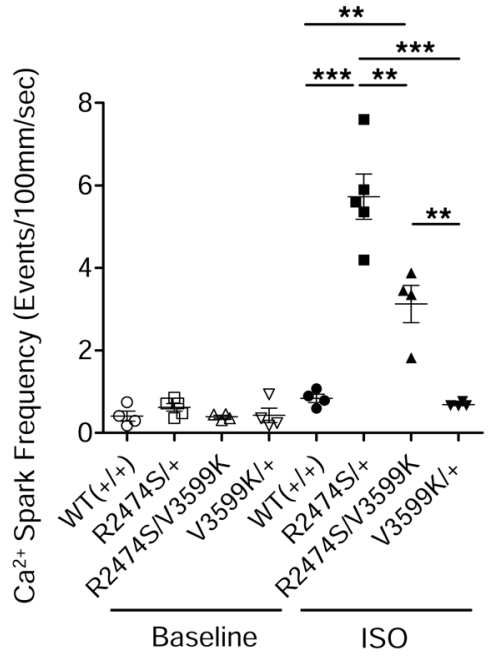

V3599K/+
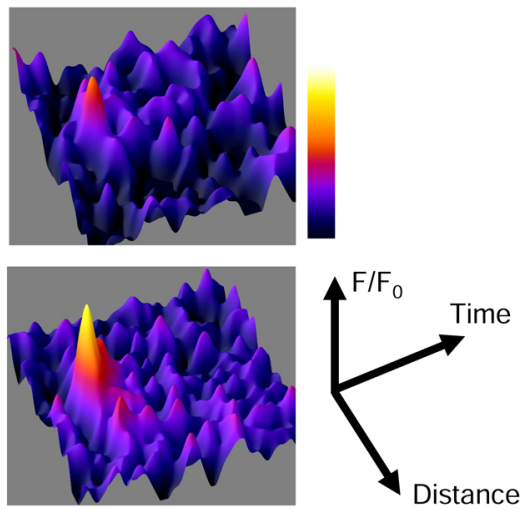

Figure 6. $\mathrm{Ca}^{2+}$ sparks in intact cardiomyocytes. (A) Representative recordings of spontaneous $\mathrm{Ca}^{2+}$ sparks. At left, recordings of line-scan images of fluo-4 AM fluorescence with ISO (10 nM) or without ISO in intact WT and KI cardiomyocytes; at right, the summarized data. Values for individual mice are plotted with mean $\pm \mathrm{SEM}$. ${ }^{* *} P<0.01$, and ${ }^{* *} P<0.001$ (1-way ANOVA with post hoc Tukey's multiple-comparison test); $n=21-35$ cells from 3 to 4 hearts. (B) Representative 3-dimensional images of $\mathrm{Ca}^{2+}$ sparks. The $\mathrm{Ca}^{2+}$ sparks were shown 3-dimensionally in intact WT and $\mathrm{KI}$ cardiomyocytes.

labeled with Alexa Fluor 488 (Alexa-CaM), into saponin-permeabilized CPVT-KI and WT cardiomyocytes. By this method, we previously confirmed that the fluorescence signal of exogenously introduced Alexa-CaM and that of immunostaining of RyR2 showed a periodical pattern associated with that of sarcomeres, and importantly the staining patterns of both CaM and RyR2 showed an excellent match with each other (Figure 2 in ref. 9). According to the method by Yang et al. (17), we first pretreated the cells with suramin $(10 \mu \mathrm{M})$ (followed by washing off) to completely abolish endogenous $\mathrm{CaM}$ and observed the time course of Alexa$\mathrm{CaM}(100 \mathrm{nM})$ binding along $\mathrm{Z}$ lines and determined the maximum specific binding (Bmax) and $\tau$ of the curves (Figure 10). Only in R2474S/WT cardiomyocytes, the Bmax of the Alexa-CaM binding decreased in the presence of cAMP, with no significant change in $\tau$. In contrast, there was no difference in the Bmax and $\tau$ between WT, R2474S/V3599S, and V3599K/+ cardiomyocytes, regardless of the presence of cAMP. These data consistently support the notion that the CaM-binding affinity to the RyR2 is reduced by R2474S mutation, and V3599K mutation can rescue the reduced CaM-binding affinity.

Direct binding of exogenous CaM to the RyR2 was also evaluated by attaching a UV cross-linker to CaM. Upon cAMP-mediated phosphorylation at Ser2808, the binding affinity of CaM to the RyR2 markedly decreased in R2474S/+-KI hearts and was restored in R2474S/V3599K-KI hearts (Figure 11A), although the phosphorylation level at Ser2808 was similarly increased by cAMP among all mice (Supplemental Figure 7). To semiquantitatively evaluate the stoichiometry of CaM binding to RyR2, 
Table 2. Effect of isoproterenol on $\mathrm{Ca}^{2+}$ spark characteristics in intact cardiomyocytes

\begin{tabular}{|c|c|c|c|c|c|}
\hline$(-)$ & $\mathrm{R} 24745 /+(n=3)$ & $1.19 \pm 0.01$ & $1.53 \pm 0.10$ & $36.17 \pm 4.36$ & 32 \\
\hline$(-)$ & V3599K/+ $(n=3)$ & $1.18 \pm 0.01$ & $1.67 \pm 0.15$ & $34.02 \pm 3.22$ & 35 \\
\hline$(+)$ & WT $(+/+)(n=3)$ & $1.27 \pm 0.02$ & $1.81 \pm 0.09$ & $36.46 \pm 2.89$ & 65 \\
\hline$(+)$ & $\mathrm{R} 2474 \mathrm{~S} /+(n=3)$ & $1.25 \pm 0.01$ & $2.33 \pm 0.06^{A}$ & $81.14 \pm 3.49^{A}$ & 352 \\
\hline
\end{tabular}

${ }^{A} P<0.01$ vs. WT (+/+) with ISO. ${ }^{B} P<0.05$ vs. WT (+/+) with ISO. ${ }^{C} P<0.01$ vs. R2474S/+ with ISO. ${ }^{D} P<0.05$ vs. R2474S/+ with ISO. $n$, the number of hearts; FWHM, full width at half maximum; FDHM, full duration at half maximum.

we used suramin again to pharmacologically displace CaM from RyR2 (15-17). First, we confirmed the concentration of suramin was enough to completely displace CaM from all 4 subunits of the RyR2. As shown in Figure 11B, suramin $(10 \mu \mathrm{M})$ almost completely inhibited the exogenous CaM binding to the RyR2 in WT hearts. Then, we observed the inhibitory effect of suramin in KI hearts. Suramin almost completely inhibited the exogenous CaM binding to the RyR2 in all KI mice, regardless of phosphorylation or mutation (Figure 11B and Supplemental Figure 8).

\section{Discussion}

Here, we demonstrated that a single amino acid substitution within the CaM-binding domain of the RyR2, by which the binding affinity of CaM to the RyR2 was markedly enhanced, inhibited $\mathrm{Ca}^{2+}$ leak from the SR, thereby inhibiting CPVT.

Liu et al. recently reported that gene transfer of a mutated CaM (GSH-M37Q), which exhibited a slowed N-terminal $\mathrm{Ca}$ dissociation rate and prolonged RyR2 refractoriness, reduced aberrant diastolic $\mathrm{Ca}^{2+}$ release and alleviated arrhythmias in a calsequestrin-associated CPVT model (20). These findings support the important role of CaM in regulating the RyR2 in the pathogenesis of CPVT. However, because CaM interacts with many other proteins in the cell and acts as a regulator or an effector molecule in a variety of cellular functions, it remains to be clarified as to whether direct association of CaM with the RyR2 really modifies the $\mathrm{Ca}^{2+}$ handling in the pathogenesis of CPVT, particularly independent of any possible action of $\mathrm{CaM}$ on other proteins. In this regard, the important aspect of this study is that to enhance the binding affinity of CaM, a single amino acid substitution was made only to the CaM-binding site within the RyR2 (V3599K), not to CaM itself. Therefore, without taking any off-target effects into consideration, we can clearly mention that the proper association of CaM to the RyR2 is essential for the prevention of the CPVT phenotype.

One of the major hypotheses to explain the RyR2 dysfunction in heart failure and lethal arrhythmias, such as CPVT, is that defective interaction between the N-terminal (N: 1-600) and the central (C: 2000-2500) domains $(4,5)$ causes an instability of the channel. Namely, in the resting state, the N-terminal and central RyR2 domains interact with each other to act as a regulatory switch that influences RyR channel gating. This tight interdomain interaction, termed "domain zipping," seems to stabilize the closed channel. Weakening of these interdomain interactions may be caused by mutations in either the N-terminal or central regions of RyR2. In support of this idea, using 3-dimensional cryoelectron microscopy, it has been reported that the $\mathrm{N}$-terminal regions within domain 5 are closely located with the central regions within domain $6(21)$, that the interaction between the $\mathrm{N}$-terminal and central mutation regions is an intersubunit (not intrasubunit) interaction (22), and that the 3-dimensional location of the Ser2808 PKA phosphorylation site was located near the domain-domain boundary (23), suggesting that PKA hyperphosphorylation at Ser2808 may alter the domain-domain interactions between the N-terminal and the central regions of the RyR2 and thus the gating properties of the channel. As a matter of fact, we previously reported that PKA phosphorylation at Ser2808 induced domain unzipping and aberrant $\mathrm{Ca}^{2+}$ release only in CPVT-associated R2474S/+-KI (but not WT) mice (6). Furthermore, accumulated evidence has recently indicated that domain unzipping is allosterically linked to reduced affinity of CaM 

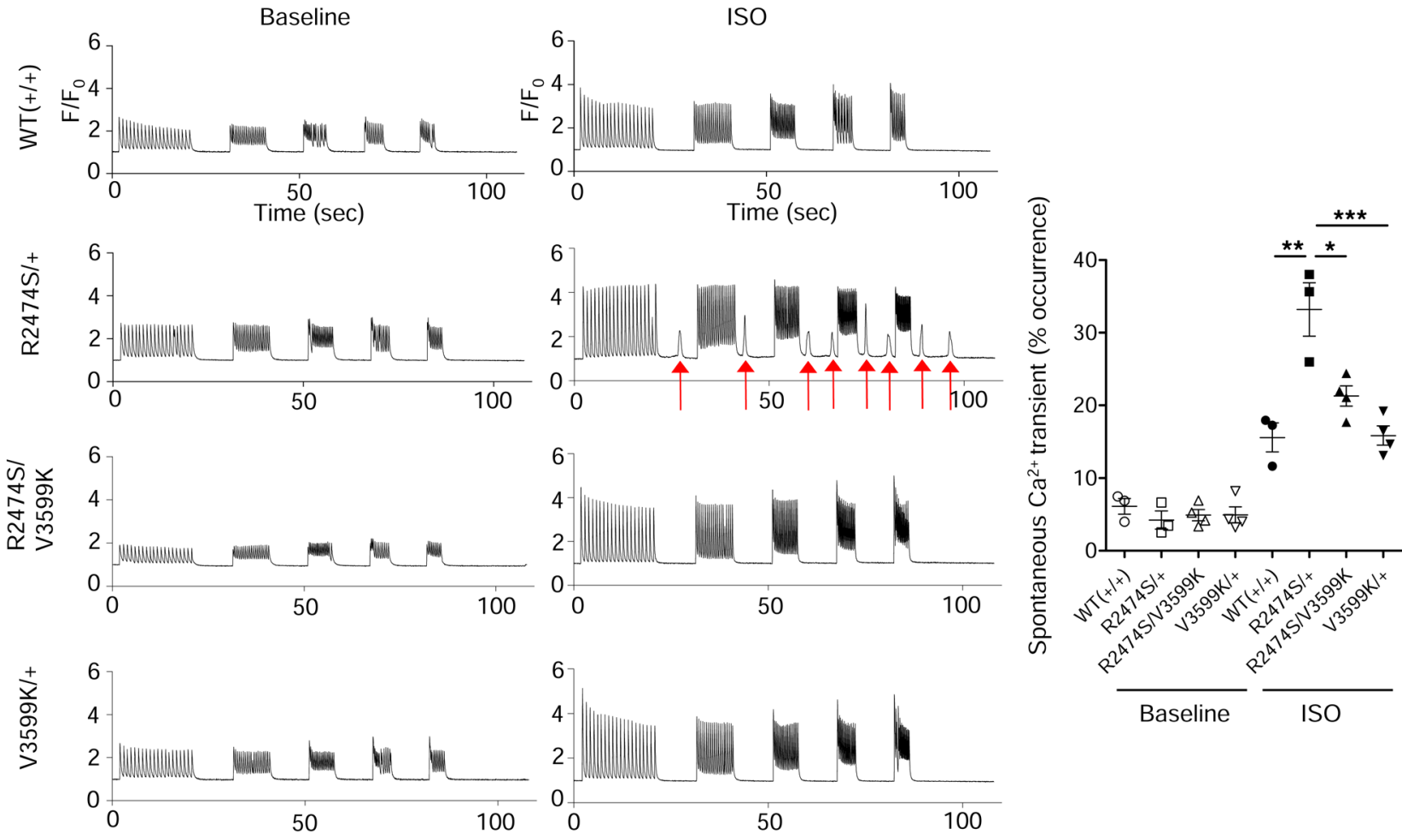

Figure 7. SCaTs in intact cardiomyocytes. (Left) Representative traces of SCaTs and (right) the summarized data as percentage occurrence of SCaTs in 874 to 1693 cells from 3 to 4 hearts in isolated intact cardiomyocytes. Arrows indicate SCaTs. The SCaTs were induced after cessation of pacing at 1, 2, 3 , 4 , and $5 \mathrm{~Hz}$ of stimulation with or without $10 \mathrm{nM}$ ISO. SCaT incidence was much higher in R2474S/+-KI cells than in WT or V3599K/+-KI cells in the presence of the ISO $(10 \mathrm{nM})$. However, SCaT incidence was markedly decreased in R2474S/V3599K-KI cells. Values for individual mice are plotted with mean \pm SEM. ${ }^{*} P<0.05,{ }^{* *} P<0.01$, and ${ }^{* *} P<0.001$ (1-way ANOVA with post hoc Tukey's multiple-comparison test).

bound to the RyR2, thereby causing aberrant diastolic $\mathrm{Ca}^{2+}$ release as a common pathogenic mechanism underlying CPVT and heart failure (10-13, 24-26).

On the basis of the above-mentioned structural background regarding the pathogenic mechanism of RyR2-associated CPVT, we propose a hypothetical model to explain why CaM was not displaced from the R2474S-mutated subunits by a single amino acid substitution (V3599K) applied against the subunits not harboring R2474S mutation (Figure 12). Because the difference in the extent of CaM binding between WT or R2474S/V3599K-KI hearts and the R2474S/+-KI hearts (with cAMP) was approximately half of that in the presence and absence of suramin in WT hearts (Figure 11B), it is suggested that in R2474S/+-KI hearts the defective intersubunit interaction, namely domain unzipping, that occurred in the R2474S-mutated subunits may allosterically decrease the binding affinity of CaM to the R2474S-mutated subunits and in turn displace approximately half of the bound CaM from the RyR2. On the other hand, in R2474S/V3599-KI hearts the defective intersubunit interaction would be minimized owing to the strong binding of CaM to the V3599K-mutated subunits, thereby preventing the decrease in the binding affinity of CaM to the remaining R2474S-mutated subunits and subsequent displacement of CaM from the RyR2. This postulated model is supported by the previous finding obtained by fluorescence resonance energy transfer method that the domain switch and CaM-binding regions are distinct but interact allosterically (rather than orthosterically) (26).

Dantrolene, which directly binds to the N-terminal domain (amino acid residues 600-610) of the RyR2, restored the domain zipping state in catecholamine-added R2474S/+-KI and failing hearts and induced the reassociation of $\mathrm{CaM}$ to the RyR2, thereby inhibiting $\mathrm{Ca}^{2+}$ leak, $\mathrm{DaD}$, and lethal arrhythmia $(6,9,11,27)$. We previously showed using a CPVT-type-KI mouse model that a single amino acid mutation (N4104K) within the CaM-like domain (CaMLD) observed in patients with CPVT causes an abnormally tight interaction between the CaMLD and the CaM-binding domains (CaMBDs), thereby decreasing the affinity of CaM to CaMBDs and in turn causing $\mathrm{Ca}^{2+}$ leak and lethal arrhythmia (28). In this $\mathrm{KI}$ mouse model, dantrolene did not inhibit the $\mathrm{Ca}^{2+}$ leak, but the increase in the affinity of $\mathrm{CaM}$ to 
A
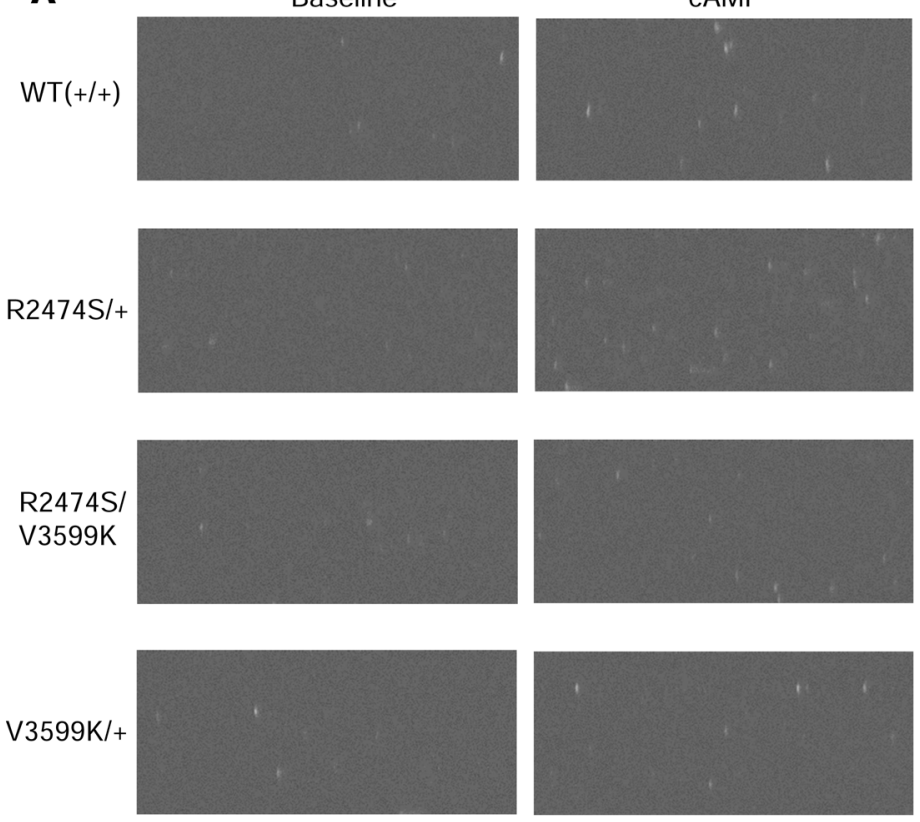

CAMP
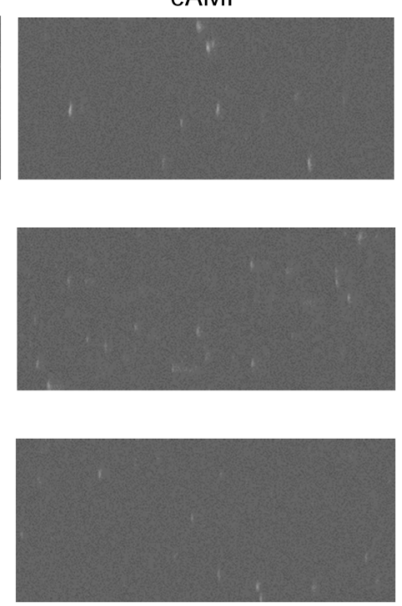

B

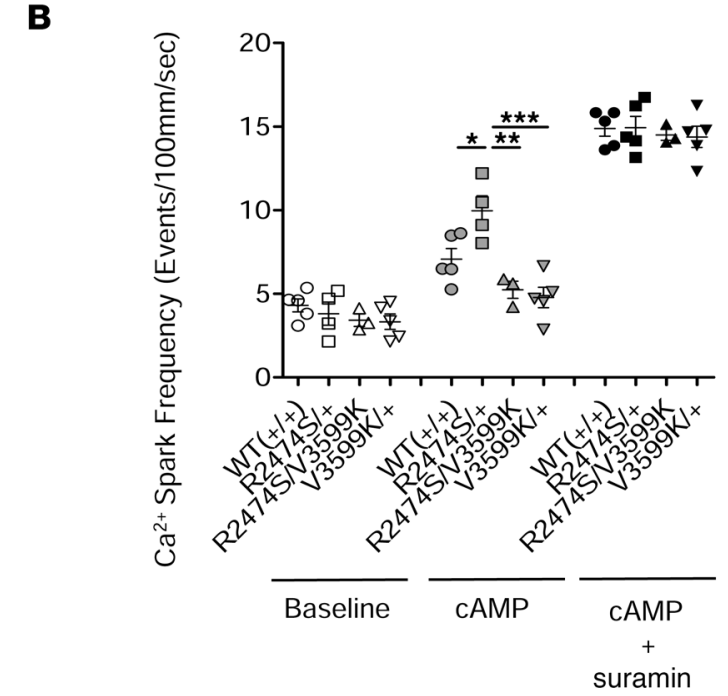

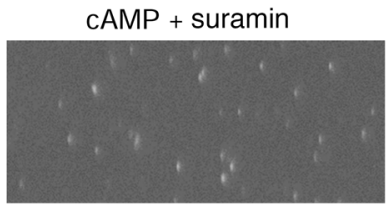
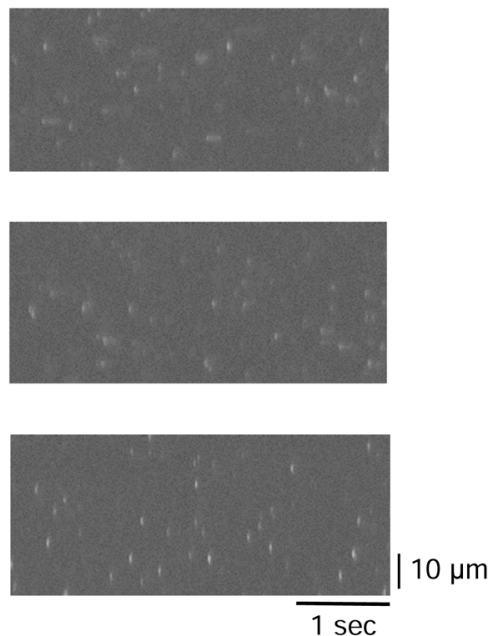

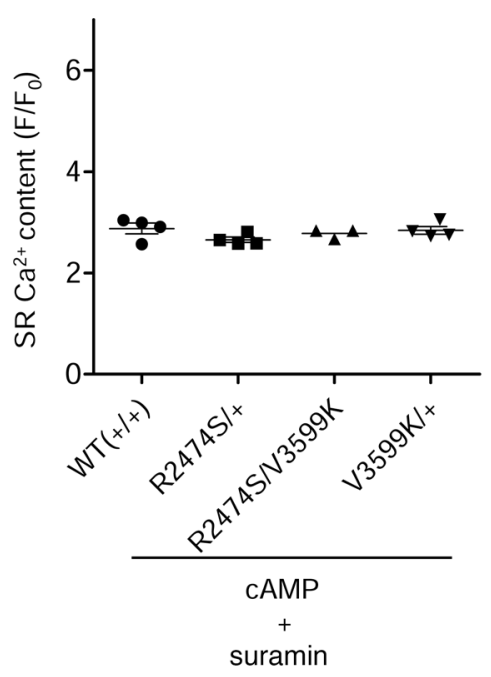

Figure 8. $\mathrm{Ca}^{2+}$ sparks in saponin-permeabilized cardiomyocytes. (A) Representative recordings of line-scan images of fluo-4 AM fluorescence (left) in the presence of exogenous CaM (100 nM) with or without cAMP $(1 \mu \mathrm{M})$ or (right) with CAMP (1 $\mu$ M) plus suramin (10 $\mu$ M). (B) Summarized data for $\mathrm{Ca}^{2+}$ spark frequency and SR $\mathrm{Ca}^{2+}$ content. To measure SR $\mathrm{Ca}^{2+}$ content, caffeine-induced $\mathrm{Ca}^{2+}$ transients were recorded by adding $20 \mathrm{mM}$ caffeine. Values for individual mice are plotted with mean $\pm \mathrm{SEM}$. ${ }^{*} P<0.05,{ }^{* *} P<0.01$, and ${ }^{* *} P<0.001$ (1-way ANOVA with post hoc Tukey's multiple-comparison test); $n=27-42$ cells from 3 to 5 hearts.

the RyR (GSH-CaM) inhibited it (28). This notion supports the ideas that dissociation of CaM from the RyR2, induced either by defective N-terminal and central domain interaction (unzipping) or by defective CaMLD and CaMBD interaction, is a common abnormal pathway leading to $\mathrm{Ca}^{2+}$ leak and that the therapeutic effect of dantrolene on the aberrant $\mathrm{Ca}^{2+}$ release is indeed $\mathrm{CaM}$ dependent. In support of this idea, Oo et al. recently showed that in single-channel recording, dantrolene caused inhibition of the RyR 1 and RyR2 only after adding physiological CaM (100 nM) but not in the absence of CaM (29).

There is a limitation of the study. It was not feasible to create double mutations (R2474S and V3599K) in the same subunit of the RyR2 by natural mating. Therefore, further study is needed to clarify whether the double mutations in the same subunit indeed restore the stability of the channel. 
A
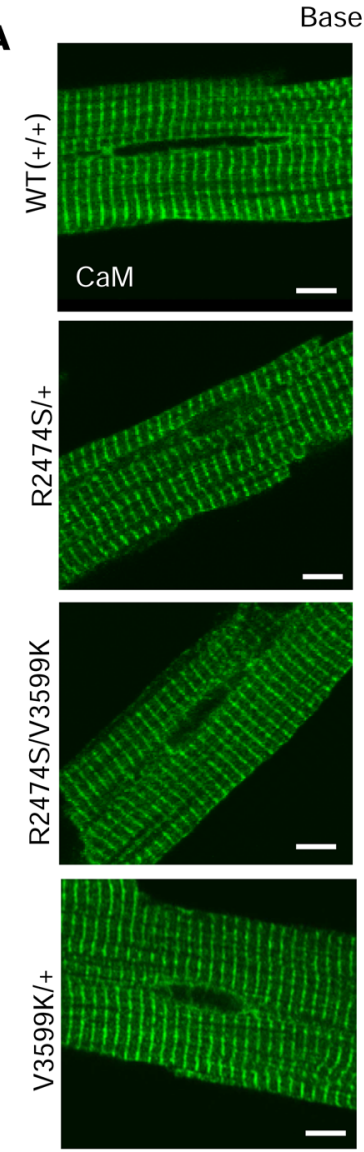

Baseline
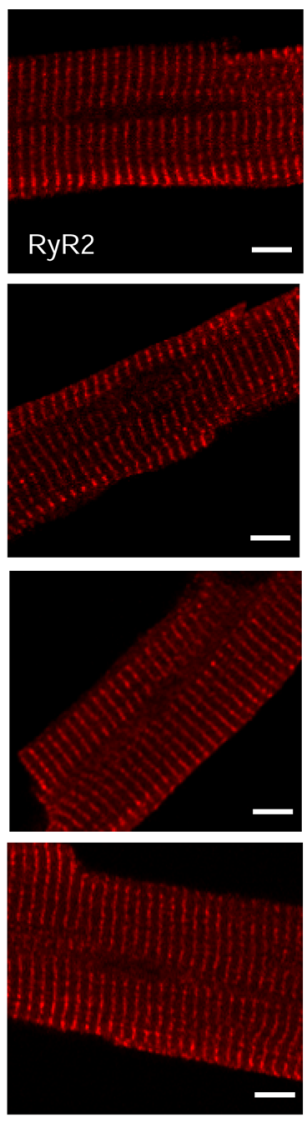

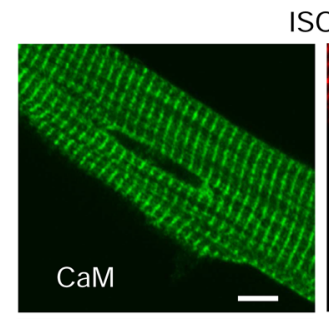

$\mathrm{SO}$
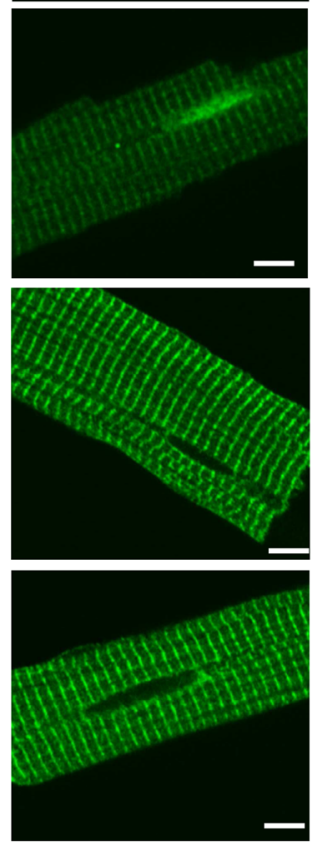

RyR2
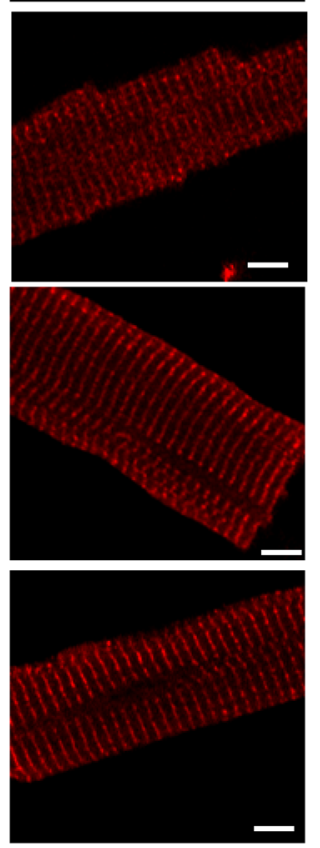

B

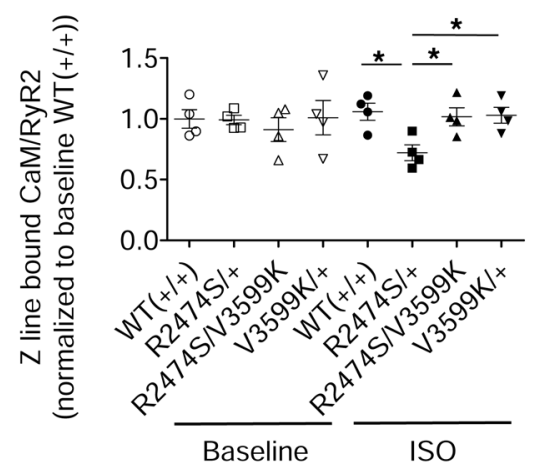

Figure 9. Localization and the binding characteristics of the endogenous CaM in intact WT and KI cardiomyocytes. (A) Representative images of the endogenous CaM, which is colocalized with the RyR2 in WT cardiomyocytes, in the (left) absence or (right) presence of ISO (10 nM); immunostaining of CaM (shown in green); immunostaining of the RyR2 (red). Scale bars: $10 \mu \mathrm{m}$. (B) Summarized data of the Z line-bound CaM and the nuclear CaM. The immunofluorescence signal of the $Z$ line-bound CaM was divided by that of the RyR2, normalized to control (baseline of WT), and expressed as a ratio. The immunofluorescence signal of the nuclear CaM was divided by that of DAPI for nuclear staining, normalized to control (baseline of WT), and expressed as a ratio. Data are presented as mean \pm SEM of 37 to 50 cells from 4 hearts. ${ }^{*} P<0.05$ (1-way ANOVA with post hoc Tukey's multiple-comparison test).

In conclusion, enhancement of the CaM-binding affinity of the RyR2 is essential to prevent CPVT-associated arrhythmogenesis. Thus, CaM may act as an intrinsic natural stabilizer for the RyR2 channel function, and hence increasing the affinity of CaM to the RyR2 could be a potent therapeutic target for heart failure and lethal arrhythmia. 
A
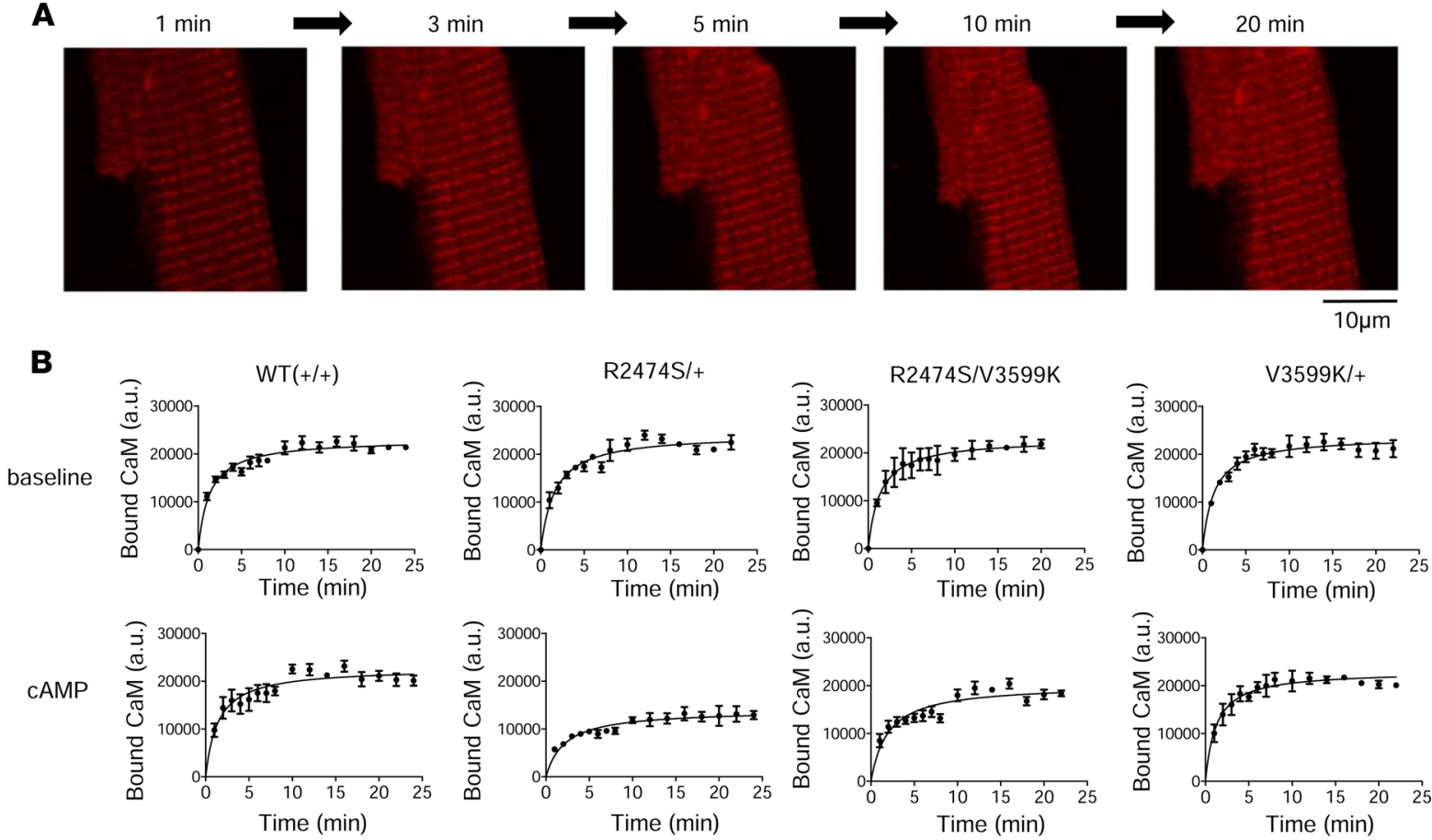

C
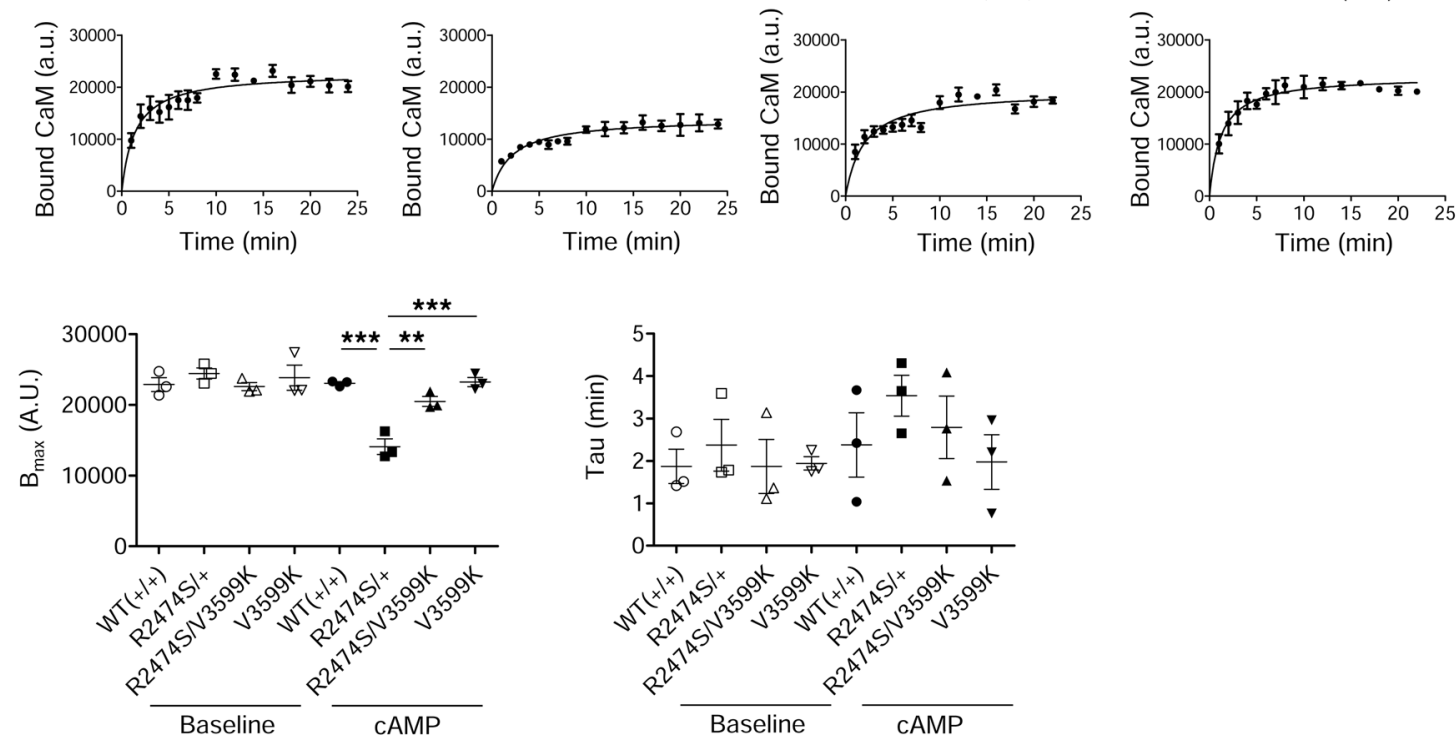

Figure 10. Kinetics of the exogenous CaM binding to the $Z$ line in cardiomyocytes. (A) Confocal images of saponin-permeabilized myocytes incubated with CaM, fluorescently labeled with Alexa Fluor 488 (Alexa-CaM; $100 \mathrm{nM}$ ), after pretreatment with suramin ( $5 \mu$ M) followed by washing off. Each image represents the time course after the incubation with CaM. (B) Time course of Alexa-CaM binding along the $Z$ line in cardiomyocytes, with or without cAMP $(1 \mu \mathrm{M})$. (C) Summarized data for Bmax and $\tau$. Values for individual mice are plotted with mean \pm SEM. ${ }^{* *} P<0.01$, and ${ }^{* * *} P<0.001$ (1-way ANOVA with post hoc Tukey's multiple-comparison test); $n=4-6$ cells from 3 hearts.

\section{Methods}

Animals. C57BL/6 mice, 20-25 weeks old, were used in this study. WT C57BL/6 mice were obtained from Japan SLC Inc. V3599K-KI mice were from UNITECH Co. Ltd. Mice were assigned to each experiment randomly, and the order of the group in each experiment was decided randomly. Allocation concealment was not applicable because the genetic background of each experimental group was different and this study did not involve any testing of therapeutic agents. Measurement and analysis of the obtained data were performed by the investigators, who were blinded to the genetic information of the mice. This study conformed to the Guide for the Care and Use of Laboratory Animals published by the National Academies Press (revised 1996). The care of the animals and the protocols used were in accordance with guidelines laid down by the Animal Ethics Committee of Yamaguchi University Graduate School of Medicine.

Echocardiogram. Cardiac function was analyzed by an F37 ultrasound machine (Hitachi medical) equipped with a 7.5-MHz probe (Hitachi, UST-5413). Mice were initially anesthetized with $4 \%$ to $5 \%$ isoflurane (mixed with oxygen) and maintained with $1 \%$ to $2 \%$ isoflurane during echocardiography. 
A

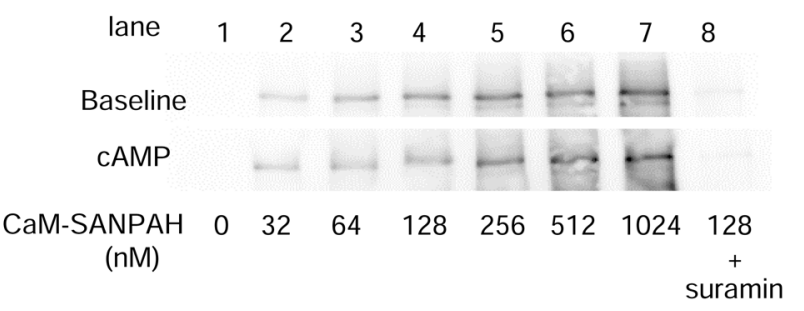

R2474S/V3599K
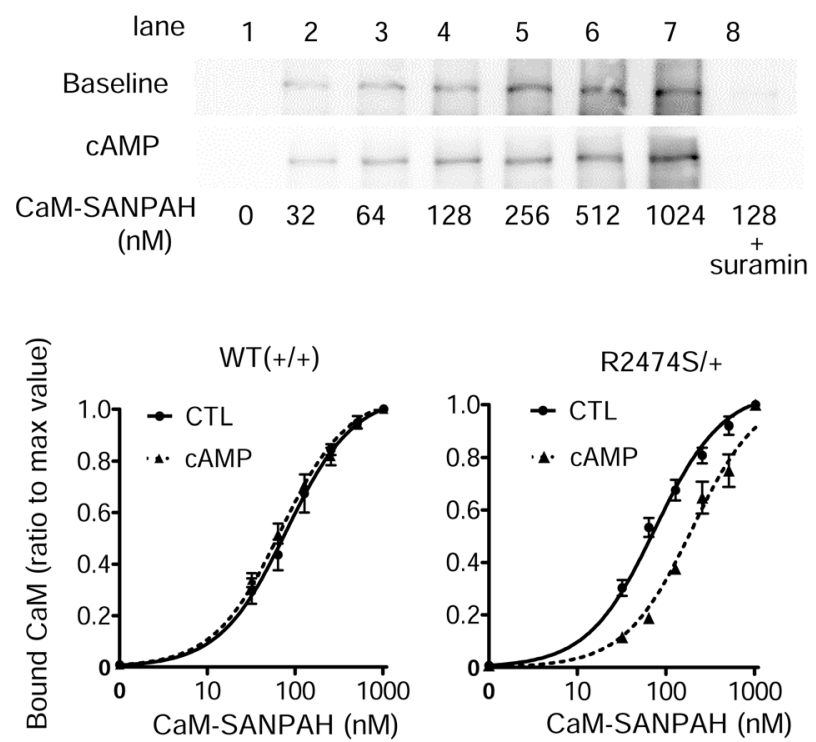

B

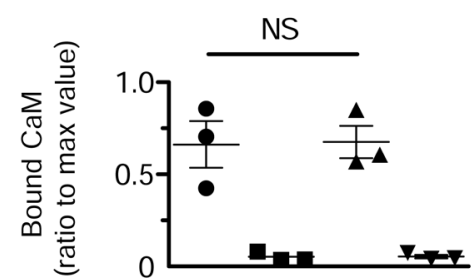

Suramin

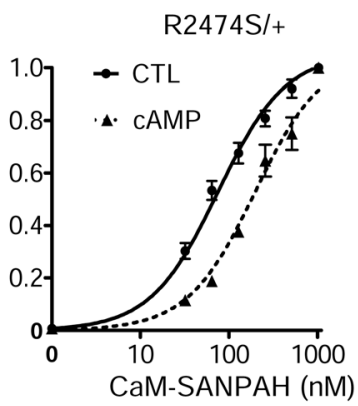

R2474S/+

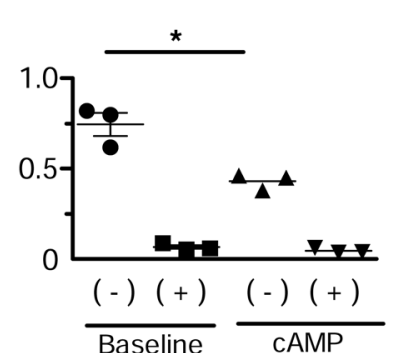

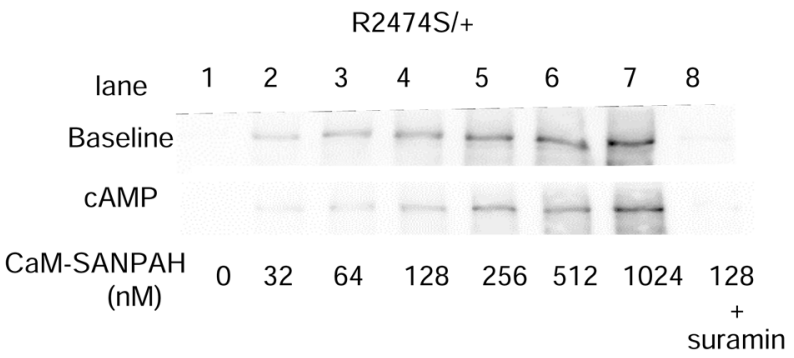

$\mathrm{V} 3599 \mathrm{~K} /+$

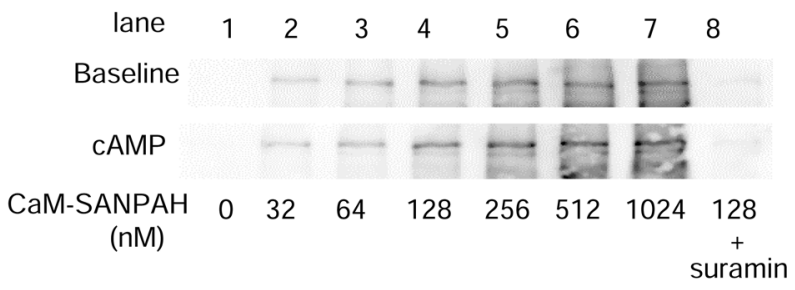

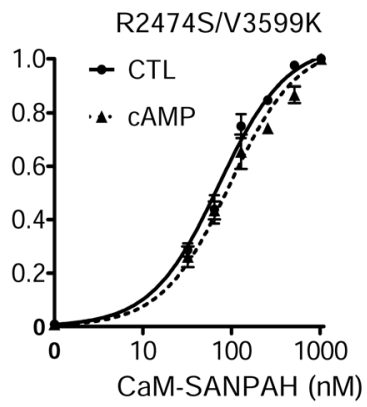

R2474S/V3599K

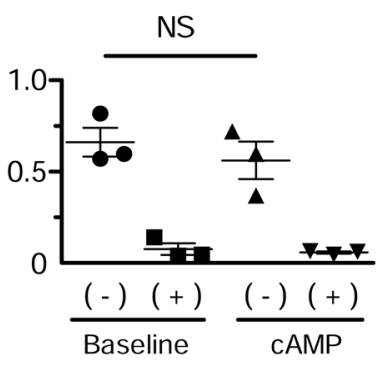

V3599K/+

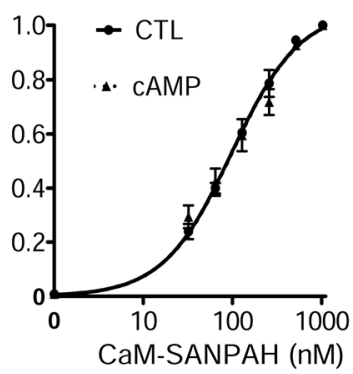

V3599K/+

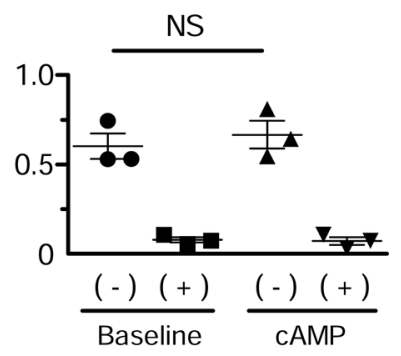

Figure 11. CaM binding to the RyR2 in cardiac homogenates. (A) (Top) Representative immunoblots of the RyR2-bound CaM-SANPAH (a photoreactive cross-linker). The WT and $\mathrm{KI}$ cardiac homogenates were first diluted in binding buffer (150 $\mathrm{mM} \mathrm{KCl,} 10 \mu \mathrm{M} \mathrm{CaCl}$, and 20 mM MES at pH 6.8) and then reacted with various concentrations of CaM-SANPAH (32-1024 nM) in the presence or absence of cAMP (1 $\mu \mathrm{M})$ and okadaic acid (1 $\mu \mathrm{M})$. CaM binding to the RyR2 was determined by immunoblotting with anti-CaM antibody to detect the RyR2-bound CaM. (Bottom) Summarized data of CaM binding to the RyR2 as a function of the concentration of CaM-SANPAH. CaM binding was expressed as the ratio to the maximum binding of CaM (at $1024 \mathrm{nM}$ ). Data are presented mean \pm SEM of 3 hearts. (B) Effect of cAMP $(1 \mu \mathrm{M})$ and suramin $(10 \mu \mathrm{M})$ on CaM binding to the RyR2. The concentration of CaM-SANPAH was fixed at 128 $\mathrm{nM}$. Data are presented as mean \pm SEM of 3 hearts. ${ }^{*} P<0.05$; NS, not significant (Student's $t$ test).

Surface ECG. ECG was monitored for the WT and the series of KI mice using ECG telemetry. Briefly, transmitters (Data Sciences International) were implanted in the back space with subcutaneous electrodes in a lead II configuration. Telemetry was first recorded with mice in a conscious state for 24 hours. After that, ECG was recorded at baseline and after the injection of epinephrine ( $1 \mathrm{mg} / \mathrm{kg}$ of body weight, i.p.) and monitored for 30 minutes in a subset of WT, R2474S/+-KI, V3599K/+-KI, and R2474S/V3599K-KI mice. Another set of WT, R2474S/+-KI, V3599K/+-KI, and R2474S/V3599K-KI mice were subjected to a treadmill exercise activity (Panlab). 


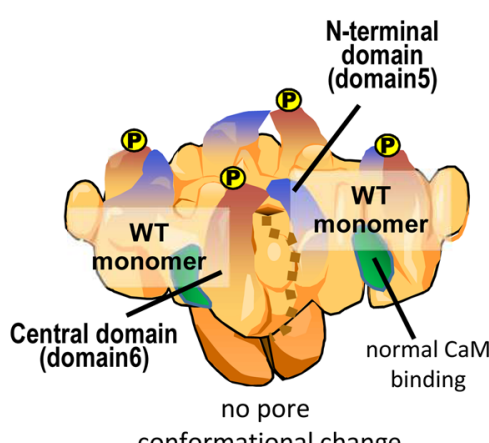

conformational change

\section{Stabilized}

RyR2 R2474S/+

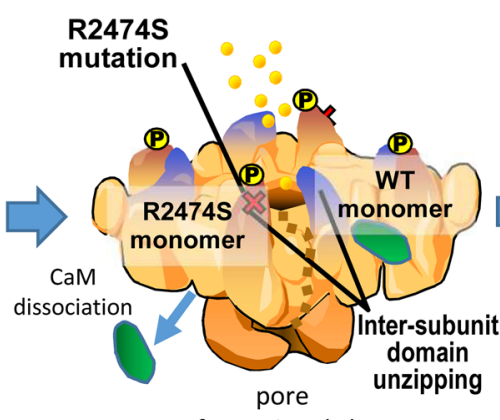

conformational change

\section{Destabilized}

RyR2 R2474S/V3599K

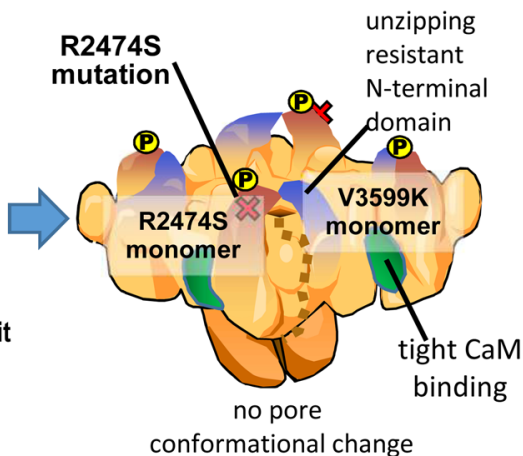

\section{Restabilized}

Figure 12. Schematic illustration of the mechanism by which V3599K substitution in RyR2 can stabilize the channel and therefore protect against $\mathrm{Ca}^{2+}$ leak. The domain unzipping in the R2474S-mutated subunits may induce a global intersubunit interaction, allosterically decreasing the binding affinity of CaM to the R2474S-mutated subunits and in turn displacing approximately half of the bound CaM from the RyR2, whereas in R2474S/ V3599-KI hearts, the postulated global intersubunit interaction followed by domain unzipping would be minimized owing to the stability of the V3599K-mutated subunits, thereby preventing the decrease in the binding affinity of CaM to the remaining R2474S-mutated subunits and subsequent displacement of CaM from the RyR2.

Isolation of cardiomyocytes. Cardiomyocytes were isolated from the mouse hearts as described previously (6) Briefly, mice were anesthetized with pentobarbital sodium (70 $\mathrm{mg} / \mathrm{kg}$ of body weight, i.p.), intubated, and ventilated with ambient air. An incision in the chest was made, and the heart was quickly removed and retrogradely perfused with a collagenase-free buffer (Thermo Fisher Scientific, DMEM, 10313) via the aorta under constant flow. The left ventricular myocardium was minced with scissors in a fresh collagenase-containing buffer, and the rod-shaped adult mouse cardiomyocytes were prepared by retrograde perfusion of the hearts with $95 \% \mathrm{O}_{2}$ and $5 \% \mathrm{CO}_{2}$-bubbled minimal essential medium (MilliporeSigma) supplemented with 50 $\mu \mathrm{M}\left[\mathrm{Ca}^{2+}\right], 0.5 \mathrm{mg} / \mathrm{ml}$ collagenase B (Roche), $0.5 \mathrm{mg} / \mathrm{ml}$ collagenase D (Roche), and $0.02 \mathrm{mg} / \mathrm{ml}$ protease type XIV (MilliporeSigma, P-5147). The $\mathrm{Ca}^{2+}$ concentration was then gradually increased to a final concentration of $1 \mathrm{mM}$ by changing the incubation medium $(50,100,300$, and $600 \mu \mathrm{M}$ and then $1 \mathrm{mM})$. The isolated mouse cardiomyocytes were transferred to laminin-coated glass culture dishes and incubated for a few hours at $37^{\circ} \mathrm{C}$ in a $5 \% \mathrm{CO}_{2}$ and $95 \% \mathrm{O}_{2}$ atmosphere.

Monitoring of $\mathrm{Ca}^{2+}$ transients of cardiomyocytes. Isolated ventricular myocytes were incubated with 20 $\mu \mathrm{M}$ fluo-4 AM (Molecular Probes) for 20 minutes at room temperature and washed twice with Tyrode's solution (made in-house; $140 \mathrm{mM} \mathrm{NaCl}, 1 \mathrm{mM} \mathrm{MgCl}$, $10 \mathrm{mM}$ glucose, $6 \mathrm{mM} \mathrm{KCl,} 5 \mathrm{mM} \mathrm{HEPES} \mathrm{pH7.4,}$ $2 \mathrm{mM} \mathrm{CaCl}_{2}$ ) as described previously (6). All experiments were conducted at $35^{\circ} \mathrm{C}$. Intracellular $\mathrm{Ca}^{2+}$ measurements with cells stimulated by a field electric stimulator (Ion Optix) were taken using a fluorescent digital microscope (Keyence, BZ9000). The relative occurrence of spontaneous $\mathrm{Ca}^{2+}$ release upon cessation of stimulation at 1, 2, 3, 4, and $5 \mathrm{~Hz}$ was measured in WT and $\mathrm{KI}$ myocytes.

Analysis of $\mathrm{Ca}^{2+}$ sparks and $\mathrm{SR} \mathrm{Ca}^{2+}$ content. The $\mathrm{Ca}^{2+}$ sparks were measured as previously described (6) using a laser-scanning confocal microscope (LSM-510, Carl Zeiss) equipped with an argon ion laser coupled with an inverted microscope (Axiovert 100, Carl Zeiss) with a Zeiss $\times 40$ oil immersion Plan-Neofluor objective (numerical aperture, 1.3; excitation at $488 \mathrm{~nm}$; emission at $>505 \mathrm{~nm}$ ). Briefly, intact cardiomyocytes were loaded with fluo-4 AM $(20 \mu \mathrm{M})$ for 20 minutes at $24^{\circ} \mathrm{C}$. Line-scan mode was used, where a single cardiomyocyte was scanned repeatedly along a line parallel to the longitudinal axis, avoiding nuclei. To monitor $\mathrm{Ca}^{2+}$ sparks, cardiomyocytes were stimulated until the $\mathrm{Ca}^{2+}$ transients reached a steady state. Stimulation was then stopped, and $\mathrm{Ca}^{2+}$ sparks were recorded during the subsequent approximately 10 seconds' rest. Data were analyzed with SparkMaster (https://sites.google.com/site/sparkmasterhome/), an automated analysis program that allows for rapid and reliable spark analysis. The variables analyzed included general image parameters (like number of detected sparks, spark frequency) as well as individual spark parameters (amplitude, FWHM, and FDHM). To assess the SR $\mathrm{Ca}^{2+}$ content, caffeine $(10 \mathrm{mM})$ was rapidly perfused to discharge the SR-loaded $\mathrm{Ca}^{2+}$. 
Immunocytochemistry analysis of endogenous RyR2-bound CaM. Isolated cardiomyocytes were fixed with 4\% paraformaldehyde (WAKO, 163-20145) in PBS for 5 minutes, washed 3 times with PBS, and permeabilized in 0.5\% Triton X-100 (MilliporeSigma) and 1\% BSA (Nacalai Tesque) for 20 minutes. Then, the cardiomyocytes were incubated overnight at $4^{\circ} \mathrm{C}$ with the anti-CaM antibody (EP799Y, Abcam) and anti-RyR antibody (MilliporeSigma, C3-33) in 1\% BSA and 0.5\% Triton X-100, followed by labeling with an Alexa Fluor 488-conjugated goat anti-rabbit (Thermo Fisher Scientific, A11008) and an Alexa Fluor 633-conjugated goat anti-mouse (Thermo Fisher Scientific, A21052) secondary antibody. The cardiomyocytes were washed 3 times with PBS.

The sarcomere-related periodical increase in the Alexa Fluor 633 and Alexa Fluor 488 fluorescence intensity from baseline was integrated with the longitudinally selected distance $(\sim 25 \mu \mathrm{m})$, and then the value was divided by the distance. Then mean value of 1 sarcomere-related increased fluorescence intensity was calculated as the arbitrary amount of RyR and RyR-bound CaM. Secondary antibody labeling alone showed no detectable fluorescence pattern.

Determination of the binding of exogenous CaM to the RyR2 in saponin-permeabilized cardiomyocytes. The exogenous CaM, fluorescently labeled with Alexa Fluor 633 (Molecular Probes), was introduced into the saponin-permeabilized WT and KI cardiomyocytes as described previously (10). Then, the distribution of localized CaM was determined by densitometric measurement of Alexa-CaM fluorescence. Briefly, the fluorescently labeled cardiomyocytes were laser-scanned with the confocal microscope (LSM-510, Carl Zeiss) (numerical aperture, 1.3; excitation at $633 \mathrm{~nm}$; emission $650 \mathrm{~nm}$ ). The sarcomere-related periodical increase in the Alexa Fluor 633 fluorescence intensity from baseline was integrated with the longitudinally selected distance $(\sim 25 \mu \mathrm{m})$, and then we divided the value by the distance.

Analysis of the binding characteristics of CaMBPs to CaM and the association of CaM to the RyR2 by CaMSANPAH cross-linking method. The binding of CaM to the RyR2 was evaluated using the photoreactive cross-linker sulfo-SANPAH (Thermo Fisher Scientific) as described previously (10). First, we made a CaM-SANPAH conjugate by mixing $50 \mu \mathrm{M}$ recombinant $\mathrm{CaM}$ in the conjugation buffer $(150 \mathrm{mM} \mathrm{KCl}$ and $20 \mathrm{mM}$ MOPS at $\mathrm{pH}$ 7.2) and $100 \mu \mathrm{M}$ sulfo-SANPAH in the dark. After 30 minutes, conjugation was quenched by adding excess amount of lysine. CaM-SANPAH conjugate was purified using Amicon Ultra (MW cutoff $10 \mathrm{kDa}$ ). Canine SR vesicles (for analysis of the binding characteristics of CaMBPs to $\mathrm{CaM}$ ) and mouse cardiac homogenates (for analysis of association of CaM to the RyR2) were diluted using the binding buffer ( $150 \mathrm{mM} \mathrm{KCl}, 10 \mu \mathrm{M} \mathrm{CaCl}_{2}$, and $20 \mathrm{mM} \mathrm{MES}$ at $\left.\mathrm{pH} 6.8\right)$ to $1 \mathrm{mg} / \mathrm{ml}$ and mixed with $100 \mathrm{nM}$ CaM-SANPAH conjugate in the dark in a glass tube with or without CaMBPs. After 10 minutes' binding time, UV irradiation was done for cross-linking. Then sample buffer was added to the cross-linked SR membrane, and Western blotting using anti-CaM antibody (Merck, MilliporeSigma) was done. CaM-SANPAH cross-linked to the RyR2 was detected with a 550-kDa band.

The binding affinity of CaM to each CaMBP was measured as an inhibition of CaM-SANPAH binding to the RyR2 in SR vesicles. When the affinity of CaM to CaMBP is higher than to the RyR2, most of the added CaM-SANPAH binds to CaMBP instead of the RyR2. To assess the conformational state of $\mathrm{CaM}$, we measured the fluorescence level of tryptophan within CaM upon its binding to CaMBPs.

CaMBP-CaM interaction measurement by QCM. Binding of CaM to WT CaMBP or V3599K CaMBP was detected by using a 27-MHz QCM (Initium Inc.), a highly sensitive mass-measuring apparatus, as we described previously (8). The QCM Au electrode was coated with CaMBP and immersed in a solution (500 $\mu \mathrm{l})$ containing $150 \mathrm{mM} \mathrm{NaCl}, 20 \mathrm{mM}$ MOPS, and $0.01 \mathrm{mM} \mathrm{Ca}^{2+}(\mathrm{pH}$ 7.2). The amount of peptide binding was determined from the frequency changes due to changes in mass on the electrode (with sensitivity of the order of subnanogram) on injection of a small volume (2-5 $\mu \mathrm{l})$ of solution containing CaM. No significant nonspecific binding of $\mathrm{CaM}$ to the Au electrode was detected.

Histology. Hearts from the WT and the series of KI mice, aged 16-22 weeks, were fixed using 10\% formalin. A complete, full-circumferential section, at the level of the 2 left ventricular papillary muscles, was selected for morphometric analysis. Hematoxylin and eosin and Masson's trichrome stains were performed for each section of the ventricle.

Immunoblot analysis. We performed immunoblot analysis of RyR2 (C3-33, MilliporeSigma), phosphorylated RyR2 at Ser2808 (p-Ser2808 RyR2), SR Ca ${ }^{2+}$-ATPase (SERCA2a, Affinity Bioreagents), phospholamban (PLB, Santa Cruz Biotechnology Inc.), phosphorylated PLB at serine 16 (p-Ser16 PLB, Fitzgerald), phosphorylated PLB at threonine 17 (p-Thr17 PLB, Santa Cruz Biotechnology Inc.), and glyceraldehyde-3-phosphate dehydrogenase (MilliporeSigma) as described previously (3). 
Statistics. Two-tailed Student's $t$ tests were used for statistical comparisons of data obtained during the 2 different situations, whereas 1-way ANOVA with a post hoc Tukey's test was used for statistical comparison of more than 2 groups. All data are expressed as mean \pm SEM. A $P$ value of less than 0.05 was considered statistically significant.

Study approval. All experiments were approved by the Animal Ethics Committee of Yamaguchi University Graduate School of Medicine.

\section{Author contributions}

YN, TY, and SK designed the research, performed experiments, analyzed data, and wrote the manuscript. YH and MT conducted the in vivo ECG recording and provocation test for VT; GF and TK assisted with the measurement of $\mathrm{Ca}^{2+}$ transients, cell shortening, and $\mathrm{Ca}^{2+}$ sparks and data analysis thereof. $\mathrm{XX}$ and SN assisted with Western blot experiments. HU, TO, and SO assisted with CaM-binding assays and data analysis thereof. MY designed and supervised the research and wrote the manuscript.

\section{Acknowledgments}

This work was supported by grants-in-aid for scientific research from the Ministry of Education, Culture, Sports, Science and Technology in Japan (17H04178 and 26293189 to MY, 15K09085 to TY, 15K09142 and 18K08039 to SK). We wish to acknowledge Yoko Okamoto and Satomi Tateda for their technical assistance.

Address for correspondence: Masafumi Yano, Department of Medicine and Clinical Science, Division of Cardiology, Yamaguchi University Graduate School of Medicine, 1-1-1 Minamikogushi, Ube, Yamaguchi 755-8505, Japan. Phone: 81.836.22.2248; Email: yanoma@yamaguchi-u.ac.jp.

1. Yano M, Yamamoto T, Kobayashi S, Matsuzaki M. Role of ryanodine receptor as a Ca2(+) regulatory center in normal and failing hearts. J Cardiol. 2009;53(1):1-7.

2. Bers DM. Macromolecular complexes regulating cardiac ryanodine receptor function. J Mol Cell Cardiol. 2004;37(2):417-429.

3. Priori SG, Chen SR. Inherited dysfunction of sarcoplasmic reticulum $\mathrm{Ca}^{2+}$ handling and arrhythmogenesis. Circ Res. 2011;108(7):871-883.

4. Yano M, Yamamoto T, Ikeda Y, Matsuzaki M. Mechanisms of Disease: ryanodine receptor defects in heart failure and fatal arrhythmia. Nat Clin Pract Cardiovasc Med. 2006;3(1):43-52.

5. Ikemoto N, Yamamoto T. Regulation of calcium release by interdomain interaction within ryanodine receptors. Front Biosci. 2002; 7:d671-d683.

6. Uchinoumi $\mathrm{H}$, et al. Catecholaminergic polymorphic ventricular tachycardia is caused by mutation-linked defective conformational regulation of the ryanodine receptor. Circ Res. 2010;106(8):1413-1424.

7. Oda $\mathrm{T}$, et al. Defective regulation of interdomain interactions within the ryanodine receptor plays a key role in the pathogenesis of heart failure. Circulation. 2005;111(25):3400-3410.

8. Yamamoto $\mathrm{T}$, et al. Identification of target domains of the cardiac ryanodine receptor to correct channel disorder in failing hearts. Circulation. 2008;117(6):762-772.

9. Kobayashi S, et al. Dantrolene, a therapeutic agent for malignant hyperthermia, markedly improves the function of failing cardiomyocytes by stabilizing interdomain interactions within the ryanodine receptor. J Am Coll Cardiol. 2009;53(21):1993-2005.

10. Xu X, et al. Defective calmodulin binding to the cardiac ryanodine receptor plays a key role in CPVT-associated channel dysfunction. Biochem Biophys Res Commun. 2010;394(3):660-666.

11. Ono $\mathrm{M}$, et al. Dissociation of calmodulin from cardiac ryanodine receptor causes aberrant $\mathrm{Ca}(2+)$ release in heart failure. Cardiovasc Res. 2010;87(4):609-617.

12. Fukuda M, et al. Enhanced binding of calmodulin to RyR2 corrects arrhythmogenic channel disorder in CPVT-associated myocytes. Biochem Biophys Res Commun. 2014;448(1):1-7.

13. Hino A, et al. Enhanced binding of calmodulin to the ryanodine receptor corrects contractile dysfunction in failing hearts. Cardiovasc Res. 2012;96(3):433-443.

14. Yamaguchi N, Takahashi N, Xu L, Smithies O, Meissner G. Early cardiac hypertrophy in mice with impaired calmodulin regulation of cardiac muscle Ca release channel. J Clin Invest. 2007;117(5):1344-1353.

15. Papineni RV, O'Connell KM, Zhang H, Dirksen RT, Hamilton SL. Suramin interacts with the calmodulin binding site on the ryanodine receptor, RYR1. J Biol Chem. 2002;277(51):49167-49174.

16. Hill AP, Kingston O, Sitsapesan R. Functional regulation of the cardiac ryanodine receptor by suramin and calmodulin involves multiple binding sites. Mol Pharmacol. 2004;65(5):1258-1268.

17. Yang Y, et al. Cardiac myocyte Z-line calmodulin is mainly RyR2-bound, and reduction is arrhythmogenic and occurs in heart failure. Circ Res. 2014;114(2):295-306.

18. Wu X, Bers DM. Free and bound intracellular calmodulin measurements in cardiac myocytes. Cell Calcium. 2007;41(4):353-364

19. Oda T, et al. Nuclear translocation of calmodulin in pathological cardiac hypertrophy originates from ryanodine receptor bound calmodulin. J Mol Cell Cardiol. 2018;125:87-97.

20. Liu B, et al. Gene transfer of engineered calmodulin alleviates ventricular arrhythmias in a calsequestrin-associated mouse model of catecholaminergic polymorphic ventricular tachycardia. J Am Heart Assoc. 2018;7(10):e008155. 
21. Wang R, et al. Localization of an $\mathrm{NH}(2)$-terminal disease-causing mutation hot spot to the "clamp" region in the three-dimensional structure of the cardiac ryanodine receptor. J Biol Chem. 2007;282(24):17785-17793.

22. Liu Z, et al. Dynamic, inter-subunit interactions between the N-terminal and central mutation regions of cardiac ryanodine receptor. J Cell Sci. 2010;123(pt 10):1775-1784.

23. Meng X, et al. Three-dimensional localization of serine 2808, a phosphorylation site in cardiac ryanodine receptor. $J$ Biol Chem. 2007;282(35):25929-25939.

24. Uchinoumi H, et al. CaMKII-dependent phosphorylation of RyR2 promotes targetable pathological RyR2 conformational shift J Mol Cell Cardiol. 2016;98:62-72.

25. Oda $\mathrm{T}$, et al. Oxidation of ryanodine receptor (RyR) and calmodulin enhance Ca release and pathologically alter, RyR structure and calmodulin affinity. J Mol Cell Cardiol. 2015;85:240-248

26. Oda $\mathrm{T}$, et al. In cardiomyocytes, binding of unzipping peptide activates ryanodine receptor 2 and reciprocally inhibits calmodulin binding. Circ Res. 2013;112(3):487-497.

27. Kobayashi S, et al. Dantrolene, a therapeutic agent for malignant hyperthermia, inhibits catecholaminergic polymorphic ventricular tachycardia in a RyR2(R2474S/+) knock-in mouse model. Circ J. 2010;74(12):2579-2584.

28. Nishimura S, et al. Mutation-linked, excessively tight interaction between the calmodulin binding domain and the C-terminal domain of the cardiac ryanodine receptor as a novel cause of catecholaminergic polymorphic ventricular tachycardia. Heart Rhythm. 2018;15(6):905-914.

29. Oo YW, et al. Essential role of calmodulin in RyR inhibition by dantrolene. Mol Pharmacol. 2015;88(1):57-63. 